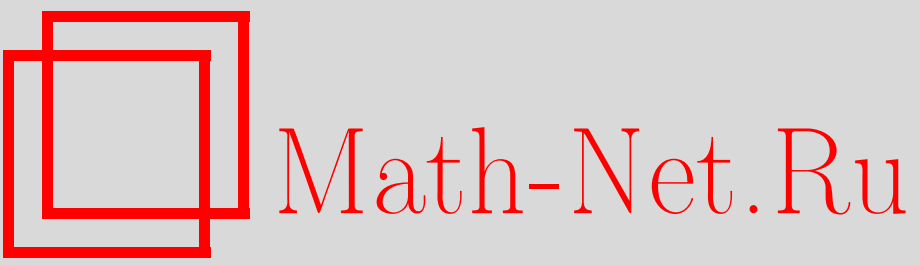

А. В. Шильков, Метод лебеговых моментов для решения уравнения переноса нейтронов, Матем. моделирование, 2020, том 32, номер 5, 59-94

DOI: https://doi.org/10.20948/mm-2020-05-04

Использование Общероссийского математического портала Math-Net.Ru подразумевает, что вы прочитали и согласны с пользовательским соглашением

http://www.mathnet.ru/rus/agreement

Параметры загрузки:

IP: 54.237 .206 .68

26 апреля 2023 г., 06:42:46 


\title{
МЕТОД ЛЕБЕГОВЫХ МОМЕНТОВ ДЛЯ РЕШЕНИЯ УРАВНЕНИЯ ПЕРЕНОСА НЕЙТРОНОВ
}

\author{
(C) 20202. \\ А.В. Шильков
}

\author{
Институт прикладной математики им. М.В. Келдыша РАН \\ ale-shilkov@yandex.ru
}

DOI: $10.20948 / \mathrm{mm}-2020-05-04$

Разработан метод лебеговых моментов для моделирования эффектов обращения резонансов, резонансной самоэкранировки, блок-эффекта в спектрах нейтронов протяженных неоднородных объектов - ядерных реакторов, защит от излучений, установок для исследования вещества. Метод использует более точную процедуру осреднения по энергии нейтронов, чем осреднение в группы. Основные составляющие метода: выделение резонансной структуры нейтронных сечений с помощью разбиения шкалы энергий на ряд множеств - носителей резонансов; выполнение лебеговского осреднения сечений и тока нейтронов в пределах носителей; разложение тока нейтронов в ряд по базисным функциям, зависящим от величины нейтронных сечений. Для расчета коэффициентов разложения (лебеговых моментов) могут применяться известные методы решения уравнения переноса нейтронов.

Ключевые слова: ядерные реакторы, нейтронная диагностика, спектр нейтронов, резонансная самоэкранировка, уравнение переноса нейтронов.

\section{LEBESGUE MOMENT METHOD FOR SOLVING THE NEUTRON TRANSPORT EQUATION}

\begin{abstract}
A.V. Shilkov
Keldysh Institute of Applied Mathematics RAS

ale-shilkov@yandex.ru

The method of Lebesgue moments for simulating the reversal of resonances, resonance self-shielding, and block effect in the neutron spectra of extended heterogeneous objects, such as nuclear reactors, radiation shielding, and installations for studying the properties of matter, is developed. The method uses a more accurate averaging procedure over neutron energy than the group averaging. The main components of the method are the refinement of the resonance structure of neutron cross sections by dividing the energy scale into a series of sets called carriers of resonances, Lebesgue averaging of cross sections and neutron flux within carriers, and the expansion of the neutron flux in a series in basis functions that depend on the magnitude of the neutron cross sections. The expansion coefficients (the so-called Lebesgue moments) can be calculated by any available method for solving the neutron transport equation.
\end{abstract}

Key words: nuclear reactors, neutron diagnostics, neutron spectrum, resonance selfshielding, neutron transport equation. 


\section{Введение}

Цель статьи состоит в изложении метода лебеговых моментов, предназначенного для моделирования полей нейтронов в неоднородных гетерогенных объектах, в том числе резонансной самоэкранировки тока нейтронов в глубине зон, содержащих однородный материал, и блок-эффекта - уменьшения самоэкранировки вблизи границ зон, содержащих разные материалы. Метод имеет три важные составляющие:

1) выделение резонансной структуры нейтронных сечений с помощью разбиения шкалы энергий $0<E<\infty$ на носители резонансов - аналоги групп, которые могут состоять не из одного, а из серии интервалов;

2) лебеговское осреднение уравнения переноса нейтронов по энергии в пределах носителей резонансов;

3) разложение тока нейтронов по базисным функциям, зависящим от величины нейтронного сечения.

Будет показано, что разбиение энергий нейтронов на носители резонансов позволяет приближенно разделить переменные $E$ и $(\mathbf{r}, t)$, от которых зависят нейтронные сечения и ток нейтронов. Лебегово осреднение монотонизирует сечения и ток с помощью замены энергии нейтронов на новую переменную - меру лебеговых множеств. Множества строятся независимо внутри каждого носителя резонансов. Выигрыш от объединения энергетических точек в одну расчетную точку примерно равен числу резонансов на носителе и может достигать двух порядков. Разложение тока нейтронов по базисным функциям спектра задает оптимальную конечную сетку расчетных точек, описывающую эволюцию поля нейтронов в объекте с минимальной (в определенном смысле) ошибкой. Кроме того, разложение позволяет приближенно восстанавливать спектр нейтронов, утерянный в результате лебеговского осреднения. Название лебеговы моменты обозначает зависимость базисных функций разложения от величины полного сечения взаимодействия нейтронов с веществом.

Процедура лебеговского осреднения (второй пункт выше) имеет дискретных родственников - метод суммы экспонент для аппроксимации функций пропускания [1], метод подгрупп [2,3], «probability table» метод [4], «multiband» метод [5,6]. В отличие от перечисленных, в методе лебеговского осреднения [7-9] вначале выполняется процедура выделения носителей резонансов, а затем выполняется переход от интегрирования функций по Риману к интегрированию по Лебегу. Получаемое в результате осредненное уравнение переноса частиц зависит от непрерывной лебеговой переменной. Но еще более важно то, что уравнение является «сквозным» для 
объекта, в котором нейтронные сечения могут иметь разрывы в пространстве. Уравнение не требует постановки дополнительных граничных условий (в дополнение к условиям на внешних границах), описывающих переходы нейтронов между подгруппами материалов на границах раздела материалов в целях сохранения непрерывности тока нейтронов через границы. Наличие подобных условий сильно снижает эффективность, например, метода подгрупп. Лебегово осреднение излагалось в предыдущих статьях автора [7-9] одновременно для переноса фотонов и нейтронов. Автор счел возможным привести в тексте вариант метода, который адаптирован к задачам переноса нейтронов и скорректирован для использования в методе моментов. Ряд вопросов, например, осреднение дифференциальных сечений, рассмотрен более подробно. Эффективность метода в задачах переноса фотонов подтверждена расчетами (см. $[10,11]$ и цит. статьи). Схематичное изложение метода лебеговых моментов для переноса фотонов и нейтронов дано в [12].

В задачах переноса фотонов дискретными родственниками метода лебеговского осреднения являются метод суммы экспонент [13-15], «k-distribution» метод [16,17], «correlated-k-distribution» метод [18-21]. Более полная библиография дана в [10]. Как и метод подгрупп, методы требуют постановки внутренних условий на границах, разделяющих различные материалы объекта. Для фотонов существуют и другие методы монотонизации сечений [22-25]. В [22] получено уравнение переноса для периодической зависимости сечений от частоты. В [23] и независимо в [24] предложена замена переменных, связывающая один из косинусов направления полета и сечение фотона. В результате коэффициенты осредненного уравнения становятся монотонными функциями новой переменной, а размерность задачи понижается на единицу. (Отметим, что замена схемы интегрирования функций по Риману на схему Лебега не меняет размерность задачи.) Метод неприменим в задачах, где есть рассеяние частиц, и имеет ограничения по пространственной симметрии задачи.

Основные уравнения. В работе обсуждается вопрос - как решать линейное уравнение Больцмана для тока нейтронов

$$
\begin{aligned}
& {\left[\frac{1}{v} \frac{\partial}{\partial t}+\Omega_{i} \frac{\partial}{\partial r_{i}}+\Sigma^{t}(E, \mathbf{r}, t)\right] \varphi(E, \boldsymbol{\Omega}, \mathbf{r}, t)=q^{S}(\varphi)+q^{e x t}(\varphi),} \\
& q^{S}(\varphi)=\int_{4 \pi} \frac{d \mathbf{\Omega}^{\prime}}{2 \pi} \int_{0}^{\infty} \Sigma^{S}\left(E^{\prime}, \mathbf{r}, t\right) v^{S}\left(E^{\prime}, \mathbf{r}, t\right) W^{S}\left(E^{\prime} \rightarrow E, \eta, \mathbf{r}, t\right) \varphi\left(E^{\prime}, \mathbf{\Omega}^{\prime}, \mathbf{r}, t\right) d E^{\prime},
\end{aligned}
$$




$$
q^{e x t}(\varphi)=\sum_{\tau} \frac{\chi^{\tau}(E, \mathbf{r}, t)}{4 \pi} \lambda^{\tau} P^{\tau}(\mathbf{r}, t)+q^{r}(E, \mathbf{\Omega}, \mathbf{r}, t)
$$

Здесь $\varphi(E, \boldsymbol{\Omega}, \mathbf{r}, t)$ - ток нейтронов в единице объема-энергии-телесного угла в момент времени $t ; \boldsymbol{\Omega}(|\boldsymbol{\Omega}|=1)$ - направление полета, $v$ - модуль скорости нейтрона; $\Sigma^{t}(E, \mathbf{r}, t)\left[\mathrm{cm}^{-1}\right]$ - полное макроскопическое сечение взаимодействия с веществом для нейтрона с энергией $E[э \mathrm{~B}] ; q^{s}(\varphi)-$ источник мгновенных нейтронов или скорость «мгновенного» появления нейтронов в пучке нейтронов $\varphi(E, \boldsymbol{\Omega}, \mathbf{r}, t)$. Мгновенные нейтроны рождаются в реакциях «s» взаимодействия первичного нейтрона $\left(E^{\prime}, \boldsymbol{\Omega}^{\prime}, \mathbf{r}, t\right)$ с нуклидами вещества; $\Sigma^{S}\left(E^{\prime}, \mathbf{r}, t\right)$ - полное макроскопическое сечение реакций «S», приводящих к появлению мгновенных нейтронов, $v^{S}\left(E^{\prime}, \mathbf{r}, t\right)-$ кратность (число) мгновенных нейтронов на выходе реакций безотносительно их энергий и направлений полета; $W^{S}\left(E^{\prime} \rightarrow E, \eta, \mathbf{r}, t\right)\left[{ }^{-1} \mathrm{~B}^{-1}\right]-$ индикатриса реакций «s» (нормированное дифференциальное сечение), $\eta=\Omega \Omega^{\prime}(-1 \leq \eta \leq 1)-$ косинус угла между направлениями полета первичного нейтрона и мгновенных нейтронов; $q^{e x t}(\varphi)-$ источник сторонних нейтронов, включающий независимый (от $\varphi$ ) источник/сток нейтронов $q^{r}(E, \boldsymbol{\Omega}, \mathbf{r}, t)$ и источник запаздывающих нейтронов $\sum \chi^{\tau} \lambda^{\tau} P^{\tau} / 4 \pi$. Запаздывающие нейтроны испускают некоторые осколки деления, называемые предшественниками запаздывающих нейтронов; $\tau$ - номер группы предшественников, $\chi^{\tau}(E, \mathbf{r}, t)\left[\right.$ эВ $\left.^{-1}\right]-$ спектр запаздывающих нейтронов, $\lambda^{\tau}\left[\right.$ сек $\left.^{-1}\right]-$ средняя постоянная спонтанного распада предшественников группы $\tau$. Изменения концентраций предшественников $P^{\tau}(\mathbf{r}, t)\left[\mathrm{cm}^{-3}\right]$ описываются системой транспортных уравнений

$$
\left[\frac{\partial}{\partial t}+u_{i} \frac{\partial}{\partial r_{i}}+\lambda^{\tau}\right] P^{\tau}(\mathbf{r}, t)=\int_{4 \pi} d \boldsymbol{\Omega} \int_{0}^{\infty} v^{\tau}(E, \mathbf{r}, t) \Sigma^{f}(E, \mathbf{r}, t) \varphi(E, \boldsymbol{\Omega}, \mathbf{r}, t) d E
$$

где $v^{\tau}(E, \mathbf{r}, t) \ll 1-$ кратность (число) предшественников в группе $\tau$ на выходе реакций деления, $\mathbf{u}-$ скорость движения вещества.

Ограничения. Уравнения (1), (2) решаются совместно с начальными данными и граничными условиями, накладываемыми на пучки нейтронов, движущиеся от внешней границы Г вглубь объекта $\boldsymbol{\Omega n}<0$ :

$$
\left[\varphi\left(E, \mathbf{\Omega}, \mathbf{r}_{\Gamma}, t\right)-\varphi^{e n t}\left(E, \mathbf{\Omega}, \mathbf{r}_{\Gamma}, t\right)\right]_{\mathbf{n} \boldsymbol{\Omega}<0}=\int_{\mathbf{n} \mathbf{\Omega}^{\prime}>0} G\left(\mathbf{\Omega}^{\prime} \rightarrow \boldsymbol{\Omega}\right) \varphi\left(E, \boldsymbol{\Omega}^{\prime}, \mathbf{r}_{\Gamma}, t\right) d \mathbf{\Omega}^{\prime},
$$


где $\mathbf{n}-$ вектор внешней нормали к границе, $\varphi^{e n t}\left(E, \boldsymbol{\Omega}, \mathbf{r}_{\Gamma}, t\right)-$ ток сторонних нейтронов, прошедших сквозь границу извне, $G\left(E, \boldsymbol{\Omega}^{\prime} \rightarrow \boldsymbol{\Omega}, \mathbf{r}_{\Gamma}, t\right)$ - эффективное сечение отражения нейтронов от границы.

Суммирование сечений. Макросечение некоторой реакции или группы реакций «x» будем обозначать заглавной буквой $\Sigma^{x}$, микросечение в расчете на один нуклид с атомным номером $A$ - малой буквой $\sigma_{A}^{x}\left[\mathrm{~cm}^{2}\right]$, $N_{A}(\mathbf{r}, t)\left[\mathrm{cm}^{-3}\right]-$ концентрация нуклидов. Полное сечение $\Sigma^{t}(E, \mathbf{r}, t)$ и сечения выхода продуктов реакций есть следующие суммы

$$
\begin{gathered}
\Sigma^{t}(E, \mathbf{r}, t)=\Sigma^{c}(E, \mathbf{r}, t)+\Sigma^{S}(E, \mathbf{r}, t), \\
\Sigma^{c}(E, \mathbf{r}, t)=\sum_{A} N_{A}(\mathbf{r}, t) \sigma_{A}^{c}(E)=\sum_{y} \Sigma^{y}(E, \mathbf{r}, t)=\sum_{y, A} N_{A}(\mathbf{r}, t) \sigma_{A}^{y}(E), \\
\left\{\begin{array}{c}
1 \\
v^{s}(E, \mathbf{r}, t)
\end{array}\right\} \times \Sigma^{s}(E, \mathbf{r}, t)=\sum_{x}\left\{\begin{array}{c}
1 \\
v^{x}(E, \mathbf{r}, t)
\end{array}\right\} \Sigma^{x}(E, \mathbf{r}, t)=\sum_{x, A} N_{A}(\mathbf{r}, t)\left\{\begin{array}{c}
1 \\
v_{A}^{x}(E)
\end{array}\right\} \sigma_{A}^{x}(E)= \\
=\left\{\begin{array}{c}
1 \\
v^{f}
\end{array}\right\} \Sigma^{f}+\sum_{m=1}^{3}\left\{\begin{array}{c}
1 \\
m
\end{array}\right\} \Sigma^{n, m \times n}=\sum_{A} N_{A}(\mathbf{r}, t)\left[\left\{\begin{array}{c}
1 \\
v_{A}^{f}(E)
\end{array}\right\} \sigma_{A}^{f}(E)+\sum_{m=1}^{3}\left\{\begin{array}{c}
1 \\
m
\end{array}\right\} \sigma_{A}^{n, m \times n}(E)\right], \\
v^{\tau}(E, \mathbf{r}, t) \Sigma^{f}(E, \mathbf{r}, t)=\sum_{A} N_{A}(\mathbf{r}, t) v_{A}^{\tau}(E) \sigma_{A}^{f}(E),
\end{gathered}
$$

где $\Sigma^{c}, \sigma_{A}^{c}$ - сечения реакций захвата нейтрона, приводящих к гибели нейтрона в результате $\alpha, \beta, \gamma$-распада составного ядра; $\Sigma^{S}, \sigma_{A}^{s}$ - сечения реакций, приводящих к испусканию мгновенных нейтронов; $\Sigma^{f}, \sigma_{A}^{f}$ - сечения реакций деления ядра; $\Sigma^{n, 1 \times n}=\Sigma^{e l}+\Sigma^{i n}$ и $\sigma_{A}^{n, 1 \times n}=\sigma_{A}^{e l}+\sigma_{A}^{i n}(m=1)-$ сечения упругого $(e l)$ и неупругого (in) рассеяния нейтронов; $\Sigma^{n, m \times n}, \sigma_{A}^{n, m \times n}(m=2,3)-$ ceчения реакций умножения нейтронов; $v^{x}(E, \mathbf{r}, t), v_{A}^{x}(E)-$ кратности мгновенных нейтронов на выходе реакций $x=s, f, m \times n, \ldots ; v^{\tau}(E, \mathbf{r}, t), v_{A}^{\tau}(E)-$ кратности предшественников запаздывающих нейтронов в группе $\tau$ на выходе реакций деления $x=f$. Аналогично суммируются дифференциальные сечения

$$
\begin{gathered}
v^{S}\left(E^{\prime}, \mathbf{r}, t\right) \Sigma^{S}\left(E^{\prime}, \mathbf{r}, t\right) W^{S}\left(E^{\prime} \rightarrow E, \eta, \mathbf{r}, t\right)= \\
=\sum_{x} v^{x}\left(E^{\prime}, \mathbf{r}, t\right) \Sigma^{x}\left(E^{\prime}, \mathbf{r}, t\right) W^{x}\left(E^{\prime} \rightarrow E, \eta, \mathbf{r}, t\right)=\sum_{x, A} N_{A}(\mathbf{r}, t) v_{A}^{x} \sigma_{A}^{x}\left(E^{\prime}\right) w_{A}^{x}\left(E^{\prime} \rightarrow E, \eta\right), \\
v^{\tau}(E, \mathbf{r}, t) \Sigma^{f}(E, \mathbf{r}, t) \chi^{\tau}(E, \mathbf{r}, t) \simeq \sum_{A} N_{A}(\mathbf{r}, t) v_{A}^{\tau}(E) \sigma_{A}^{f}(E) \chi_{A}^{\tau}(E) .
\end{gathered}
$$


Здесь $W^{x}\left(E^{\prime} \rightarrow E, \eta, \mathbf{r}, t\right), w_{A}^{x}\left(E^{\prime} \rightarrow E, \eta\right)\left(\eta=\mathbf{\Omega}^{\prime}\right)-$ индикатрисы группы реакций $x ; \chi^{\tau}(E, \mathbf{r}, t), \chi_{A}^{\tau}(E)$ - спектры запаздывающих нейтронов. Все индикатрисы и спектры нормированы на единицу

$$
\begin{aligned}
& \int_{0}^{\infty} d E \int_{-1}^{1} W^{x}\left(E^{\prime} \rightarrow E, \eta, \mathbf{r}, t\right) d \eta=\int_{0}^{\infty} d E \int_{-1}^{1} w_{A}^{x}\left(E^{\prime} \rightarrow E, \eta\right) d \eta=1, \\
& \int \chi^{\tau}(E, \mathbf{r}, t) d E=\int \chi_{A}^{\tau}(E) d E=1 .
\end{aligned}
$$

Концентрации нуклидов. Если нам требуется рассматривать изменения концентраций нуклидов $N_{A}(\mathbf{r}, t)$ в пространстве-времени, то (1)-(3) дополняются связанной системой уравнений ядерных реакций

$$
\begin{gathered}
{\left[\frac{\partial}{\partial t}+u_{i} \frac{\partial}{\partial r_{i}}+\lambda_{A}+R_{A}(\mathbf{r}, t)\right] N_{A}(\mathbf{r}, t)=\sum_{z, B}\left[\xi_{B \rightarrow A}^{z} \lambda_{B \rightarrow A}^{z}+S_{B \rightarrow A}^{z}(\mathbf{r}, t)\right] N_{B}(\mathbf{r}, t),} \\
\lambda_{A}=\sum_{z, B} \lambda_{A \rightarrow B}^{z}, \quad R_{A}(\mathbf{r}, t)=\sum_{z, B} R_{A \rightarrow B}^{z}(\mathbf{r}, t), \\
\left\{\begin{array}{l}
R_{B \rightarrow A}^{z}(\mathbf{r}, t) \\
S_{B \rightarrow A}^{z}(\mathbf{r}, t)
\end{array}\right\}=\int_{4 \pi} d \mathbf{\Omega} \int_{0}^{\infty}\left\{\begin{array}{c}
1 \\
v_{B \rightarrow A}^{z}(E)
\end{array}\right\} \times \sigma_{B \rightarrow A}^{z}(E) \varphi(E, \mathbf{\Omega}, \mathbf{r}, t) d E .
\end{gathered}
$$

Здесь $\lambda_{B \rightarrow A}^{z}-$ постоянная спонтанного распада нуклида $B$ с образованием нуклида $A$ в реакции «z»; $\sigma_{B \rightarrow A}^{z}(E)$ - сечение соответствующей вынужденной реакции; $\xi_{B \rightarrow A}^{z}$ и $v_{B \rightarrow A}^{z}(E)-$ кратности нуклидов $A$ на выходе реакций.

Зависимость сечений $\sigma_{A}^{x}(E), x=t, s, f, m \times n, e l, \ldots$ от энергии содержит множество резонансов, возникающих при совпадении энергии нейтрона с разностью энергий квантовых состояний составного ядра. Вблизи резонансов сечения резко возрастают по величине, иногда на несколько порядков. Область резонансов для тяжелых ядер простирается от энергий $\sim 0.5$ э до энергий $\sim 1 \mathrm{Kэ} \mathrm{B}=10^{3}$ эВ, а для легких и средних ядер до энергий $\sim 1 \mathrm{MэB}=10^{6}{ }$ В. Число резонансов каждого ядра обычно велико, а резонансы узки. В тяжелых ядрах ширины узких резонансов имеют порядок $\sim 1 \ni \mathrm{B}$, в средних и легких ядрах - 1КэВ. Для средних ядер кроме узких резонансов иногда наблюдаются средние резонансы с ширинами $\sim 10 \div 100$ КэВ и гигантские резонансы с ширинами до $1 \mathrm{MэB.} \mathrm{С} \mathrm{увеличением} \mathrm{энергии} \mathrm{расстояние} \mathrm{между} \mathrm{резонансами}$ уменьшается и сравнивается с их шириной вблизи верхней границы области. Этот участок называется областью неразрешенных резонансов. Рис.1 демонстрирует различие в положении области резонансов для некоторых ядер. 


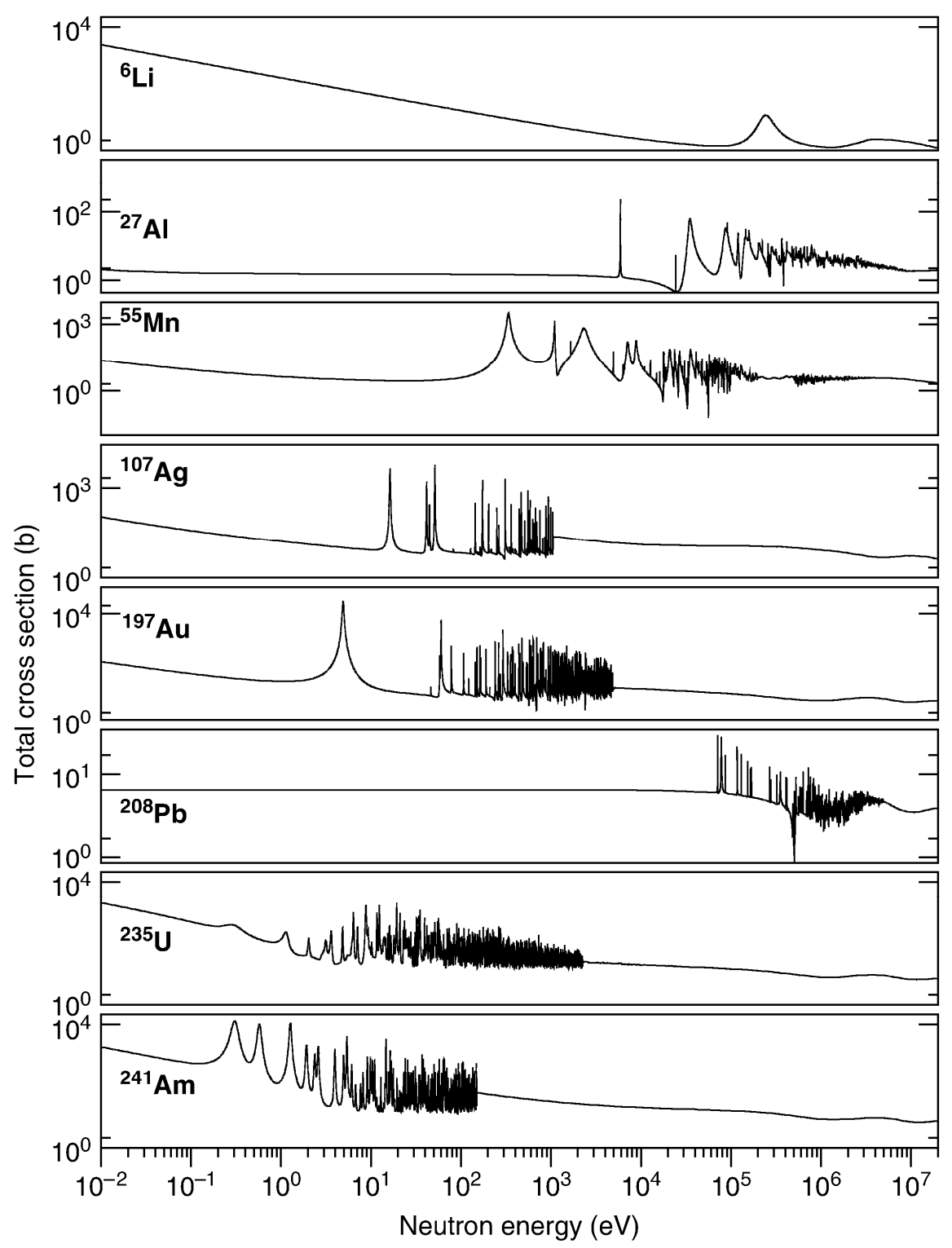

Рис.1. Полное сечение взаимодействия нейтрона с ядром (в барн, $10^{-24} \mathrm{~cm}^{2}$ ) для ряда элементов. Рисунок заимствован из [26].

Измерение параметров резонансов составляет предмет множества исследований. Результаты оцениваются [26-28] и публикуются в библиотеках ядерных данных [29]. Наиболее полные библиотеки: ENDF (Evaluated Nuclear Data Files) [30], РОСФОНД (РОСсийская библиотека Файлов Оцененных Нейтронных Данных) [31], JEF (Joined European Files) [32], JENDL (Japanies Evaluated Nuclear Data Library) [33]. Пояснение данных, формул и компьютерных программ, выполняющих реконструкцию сечений, можно найти в описаниях библиотек и в [28, 34-36].

Представление (формулы) сечений через экспериментально определяемые параметры резонансов дает теория ядерных реакций при низких энергиях $E \ll m c^{2}$. Теория достаточно полно освещена в учебниках и моногра- 
фиях. Для представления сечений применяются формулы Брейта-Вигнера, Рича-Мура, Адлер-Адлера. Приведем формулы полного сечения $\sigma_{A}^{t}$, сечения упругого рассеяния $\sigma_{A}^{e l}$ и сечений неупругих реакций $\sigma_{A}^{x}(x \neq t, e l)$ в одноуровневом приближении Брейта-Вигнера

$$
\begin{gathered}
\left\{\begin{array}{c}
\sigma_{A}^{t}(E) \\
\sigma_{A}^{x}(E)
\end{array}\right\}=\left\{\begin{array}{c}
\sigma^{p}(E) \\
0
\end{array}\right\}+4 \pi^{2} \lambda^{2} \sum_{r} \sum_{l, J} n_{l J} g_{l J} \Gamma_{r}^{e l} \times\left\{\begin{array}{c}
\theta_{r}(E) \cos 2 \phi_{l}+\chi_{r}(E) \sin 2 \phi_{l} \\
\theta_{r}(E) \Gamma_{r}^{x} / \Gamma_{r}, \quad x \neq t, e l
\end{array}\right\},(8) \\
\sigma_{A}^{e l}(E)=\sigma_{A}^{t}(E)-\sum_{x \neq e l} \sigma_{A}^{x}(E), \quad \sigma^{p}(E)=4 \pi \hbar^{2} \sum_{l, J} n_{l J} g_{l J} \sin ^{2} \phi_{l}, \\
\left\{\begin{array}{c}
\theta_{r}(E) \\
\chi_{r}(E)
\end{array}\right\}=\frac{1 / \pi}{\left[E-E_{r}\right]^{2}+\Gamma_{r}^{2}}\left\{\begin{array}{c}
\Gamma_{r} \\
E-E_{r}
\end{array}\right\}, \quad\left\{\begin{array}{l}
\theta_{r}(E) \\
\chi_{r}(E)
\end{array}\right\} d E=\left\{\begin{array}{l}
1 \\
0
\end{array}\right\}, \quad \Gamma_{r}=\Gamma_{r}^{e l}+\sum_{x \neq e l} \Gamma_{r}^{x} .
\end{gathered}
$$

Здесь $r$ - номер резонанса, квантовое число $l$ - угловой момент налетающего нейтрона, $J$ - полный спин составного ядра; $\sigma^{p}(E)$ - сечение потенциального рассеяния, $\theta_{r}(E)$ и $\chi_{r}(E)$ - симметричный и антисимметричный профили резонанса; $\lambda=\hbar / \sqrt{2 \mu E}(\mu=m A /[A+1])-$ длина волны де-Бройля, $n_{l J}(E)$ - вероятность реализации канала столкновения $(l, J), g_{l J}-$ спиновой статистический вес. Фазовый сдвиг $\phi_{l}(E)$ и полуширины резонанса $\Gamma_{r}(E)$, $\Gamma_{r}^{e l}(E), \Gamma_{r}^{x}(E)$ зависят от энергии нейтрона $E$, канала столкновения и свойств ядра. При приближении к энергии резонанса $E \approx E_{r}$ симметричный профиль резонанса $\theta_{r}(E)$ меняется быстрее остальных функций.

Зависимость дифференциальных сечений. Анализ и оценка экспериментальных данных выполняются с помощью разложения индикатрис реакций в ряд Фурье по ортогональным на отрезке $-1 \leq \eta \leq 1$ многочленам Лежандра $P_{n}(\eta)(n=0,1, \ldots)$, где $\eta=\Omega \Omega^{\prime}-$ косинус угла реакции

$$
\begin{gathered}
w_{A}^{x}\left(E^{\prime} \rightarrow E, \eta\right) \simeq \sum_{n=0}^{N} \frac{2 n+1}{2} P_{n}(\eta) w_{A, n}^{x}\left(E^{\prime} \rightarrow E\right) \\
w_{A, n}^{x}\left(E^{\prime} \rightarrow E\right)=\int_{-1}^{1} P_{n}(\eta) w_{A}^{x}\left(E^{\prime} \rightarrow E, \eta\right) d \eta, \quad \int_{0}^{\infty} w_{A, n}^{x}\left(E^{\prime} \rightarrow E\right) d E=\delta_{n 0}, \\
P_{0}(\eta)=1, P_{1}(\eta)=\eta, P_{2}(\eta)=\frac{3 \eta^{2}-1}{2}, \ldots, \quad \int_{-1}^{1} P_{n}(\eta) P_{m}(\eta) d \eta=\frac{2}{2 n+1} \delta_{m n},
\end{gathered}
$$

$w_{A, n}^{x}-$ коэффициенты разложения.

Индикатриса реакций деления нуклидов тория, урана и трансурановых 
элементов $w_{A}^{f}\left(E \rightarrow E^{\prime}, \eta\right)(x=f)$ слабо зависит от $\eta$ и энергии первичного нейтрона $E^{\prime}$ и имеет сильную зависимость от энергии мгновенных нейтронов $E$. Коэффициент разложения нулевого порядка $w_{A, 0}^{f}\left(E \rightarrow E^{\prime}\right)$ называется спектром мгновенных нейтронов деления. Нейтроны рождаются с энергиями

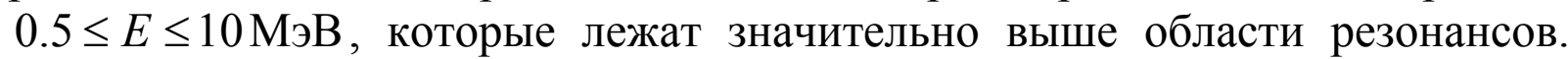
Средняя энергия спектра нейтронов деления $235 \mathrm{U}$ равна $E \approx 2 \mathrm{MэВ.} \mathrm{Все} \mathrm{спек-}$ тры нормированы на единицу:

$$
W_{0}^{f}\left(E^{\prime} \rightarrow E, \mathbf{r}, t\right)=\frac{\sum N_{A}(\mathbf{r}, t) \sigma_{A}^{f}\left(E^{\prime}\right) w_{A, 0}^{f}\left(E^{\prime} \rightarrow E\right)}{\sum N_{A}(\mathbf{r}, t) \sigma_{A}^{f}\left(E^{\prime}\right)}, \quad \int_{0}^{\infty} W_{0}^{f}\left(E^{\prime} \rightarrow E\right) d E=1 .
$$

Индикатриса упругого рассеяния нейтронов $w_{A}^{e l}\left(E^{\prime} \rightarrow E, \eta\right)(x=e l)-$ это функция с ограниченным носителем. Она сильно зависит от разности энергий $E^{\prime}-E$. Зависимость от $\eta=\Omega \Omega^{\prime}$ существенна только при рассеянии на легких ядрах. Оценочную формулу можно получить из законов сохранения энергии-импульса в предположении изотропии рассеяния в системе центра масс нейтрона и ядра [37, с.262]). При переходе в систему наблюдателя возникает жесткая зависимость между потерей энергии нейтроном и углом рассеяния $\eta_{A}^{e l}$ в системе наблюдателя, выражаемая $\delta$-функцией

$$
\begin{gathered}
w_{A}^{e l}\left(E^{\prime} \rightarrow E, \eta\right) \approx \frac{\theta(1-|\eta|)}{\alpha_{A} E^{\prime}} \delta\left(\eta-\eta_{A}^{e l}\right), \quad E^{\prime} \geq 10 T_{[\ni]]}, \\
\theta(x)=\left\{\begin{array}{c}
1, x \geq 0 \\
0, x<0
\end{array}\right\}, \quad \alpha_{A}=\frac{4 A}{[A+1]^{2}}, \quad \eta_{A}^{e l}\left(E^{\prime}, E\right)=\frac{A+1}{2}\left[\frac{E}{E^{\prime}}\right]^{1 / 2}-\frac{A-1}{2}\left[\frac{E^{\prime}}{E}\right]^{1 / 2}, \\
w_{A, n}^{e l}\left(E^{\prime} \rightarrow E\right) \approx \frac{P_{n}\left(\eta_{A}^{e l}\right)}{\alpha_{A} E^{\prime}} \theta\left(E^{\prime}-E\right) \theta\left(E-E^{\prime}+\alpha_{A} E^{\prime}\right), \quad \Delta_{A}\left(E^{\prime}\right)=\alpha_{A} E^{\prime} .
\end{gathered}
$$

$\Delta_{A}\left(E^{\prime}\right)$ - ширина носителя индикатрисы (ступеньки замедления).

Зависимость сечений от координат. Микросечения $\sigma_{A}^{x}(E)(x=t, s, c, f$, $m \times n, e l, \ldots)$ зависят от координат $(\mathbf{r}, t)$ через температуру вещества $T(\mathbf{r}, t)$. Эта зависимость в списке аргументов не указана. Тепловое движение атомов и молекул приводит к уширению узких резонансов. Соответственно, в формулах (8) (и аналогичных) выполняется свертка профилей резонансов $\theta_{r}(E), \chi_{r}(E)$ с быстроспадающим распределением Гаусса. Если температура не превышает 10 эВ, то искажения затрагивают ядра узких резонансов в пределах доплеровской ширины, оставляя неизменными крылья. Зависимость от температуры «почти всюду» является слабой. 
В отличие от микросечений, макросечения $\Sigma^{x}(E, \mathbf{r}, t) \quad(x=t, s, c, f$, $m \times n, e l, \ldots)$ могут сильно зависеть от координат $(\mathbf{r}, t)$. Объект, в котором движутся нейтроны, обычно состоит из пространственных зон, заполненных материалами, имеющими разный состав нуклидов. Концентрации нуклидов $N_{A}(\mathbf{r}, t)$ могут испытывать разрыв на границах между зонами, например, обращаться в ноль. Также концентрации нуклидов могут меняться в пространстве-времени в пределах одного материала. Например, в топливных элементах ядерного реактора со временем происходит неоднородное выгорание $235 \mathrm{U}$ вследствие эффекта самоэкранировки.

Обращение резонансов. Рассмотрим гетерогенный объект. Пусть на границах между зонами ток нейтронов $\varphi(E, \boldsymbol{\Omega}, \mathbf{r}, t)$ меняется с изменением $E$ сравнительно медленно. В глубине зоны, заполненной однородным материалом, решение уравнения (1) асимптотически стремится к стационарной точке уравнения - режиму, в котором выполняется баланс между скоростью ухода и скоростью прихода нейтронов в пучок:

$$
\varphi(E, \mathbf{\Omega}, \mathbf{r}, t) \rightarrow \varphi_{0}(E, \mathbf{r}, t)=q\left(\varphi_{0}\right) / \Sigma^{t}(E, \mathbf{r}, t) \sim F(E) / \Sigma^{t}(E, \mathbf{r}, t) .
$$

Скорость прихода нейтронов $q\left(\varphi_{0}\right)$ относительно медленно меняется по энергии $E$ в сравнении с функцией большой и резкой вариации $\Sigma^{t}(E)$. Поэтому в глубине зоны ток нейтронов $\varphi(E, \boldsymbol{\Omega}, \mathbf{r}, t)$ изрезан глубокими провалами - «обращенными резонансами» вблизи энергий резонансов $E \approx E_{r}$. Провалы приводят к снижению скоростей реакций $\int \Sigma^{x}(E) \varphi(E) d E$, в том числе реакций деления. Эффект называется резонансной самоэкранировкой поля нейтронов. Схожий эффект - «выедание» спектра в резонансах, возникает при распространении излучения граничного источника вглубь объекта.

Характер зависимостей индикатрисы деления и индикатрисы упругого рассеяния (10) от энергии нейтронов $E$ позволяет оценить $E$-зависимость функционала $q\left(\varphi_{0}\right) \sim F(E)$ [37, с.277]. Синтетический спектр $F(E)$ в приближении узких резонансов (приближении Вигнера) есть некоторая сшивка спектра нейтронов деления, спектра Ферми $\sim 1 / E$ и спектра области термализации нейтронов. В задачах расчета защиты и нейтронной диагностики спектр нейтронов деления заменяется спектром граничного источника $\varphi^{\text {ent }}$, если объект не содержит делящихся нуклидов. «Широкие» резонансы с шириной, превышающей ширину носителя индикатрисы рассеяния (см. (10)), могут вносить мелкомасштабные возмущения в синтетический спектр $F(E)$. Возмущения можно найти аналитически или с помощью вспомогательного 
численного расчета. В комплексе программ NJOY [34,35] расчет выполняется программой Flux calculator.

Асимптотический спектр $\varphi_{0}$ применяется для подготовки групповых констант [6, 34-38] методом Бондаренко [39]. Константы используются в расчетах переноса нейтронов в многогрупповом приближении [40-43].

\section{1. Разделение переменных. Носители резонансов}

Вне области резонансов, где нейтронные сечения - медленно меняющиеся функции энергии, будем пользоваться групповым осреднениием сечений и многогрупповым описанием переноса нейтронов. В области резонансов будем выполнять более точное осреднение. Уменьшим размерность задачи, объединив нуклиды в несколько компонентов.

Компонентом «С» будем называть совокупность нуклидов, концентрации которых меняются подобно друг другу в пределах объекта

$$
\frac{N_{A}(\mathbf{r}, t)}{N_{C}(\mathbf{r}, t)} \approx \mathrm{const}, \quad N_{C}(\mathbf{r}, t)=\sum_{A \subset C} N_{A}(\mathbf{r}, t), \quad \mathbf{r} \in V(t), 0 \leq t \leq T .
$$

Здесь $N_{C}$ - концентрация компонента, $V(t)$ - объем объекта, $0 \leq t \leq T-$ характерный для задачи интервал времени. Если в некоторых зонах объекта концентрации нуклидов удовлетворяют (12), а в остальных зонах концентрации близки к нулю, то нуклиды составляют компонент. В компоненты объединяются нуклиды, не участвующие в ядерных превращениях. Это легкие нуклиды замедлителей нейтронов, нуклиды конструкционных материалов (бетон, сталь, ...). Также в компоненты объединяются осколки деления и трансурановые нуклиды, возникающие в цепочках ядерных реакций в «подобных» пропорциях от нулевой или малой концентрации. Основные делящиеся нуклиды $233 \mathrm{U}, 235 \mathrm{U}, 238 \mathrm{U}, 239 \mathrm{Pu}$ и $232 \mathrm{Th}$ рассматриваются как отдельные компоненты, если их нельзя отнести к одной из групп (12). Нейтронные макросечения в разрезе компонентов имеют вид (4), (5) с заменой индекса нуклида « $A$ » на индекс компонента «C» и заменой микросечений

$$
\begin{gathered}
\left\{\begin{array}{c}
1, \quad v_{C}^{x}\left(E^{\prime}\right) \\
w_{C}^{x}\left(E^{\prime} \rightarrow E\right) v_{C}^{x}\left(E^{\prime}\right)
\end{array}\right\} \sigma_{C}^{x}\left(E^{\prime}\right)=\sum_{A \subset C} \frac{N_{A}(\mathbf{r}, t)}{N_{C}(\mathbf{r}, t)}\left\{\begin{array}{cc}
1, & v_{A}^{x}\left(E^{\prime}\right) \\
w_{A}^{x}\left(E^{\prime} \rightarrow E\right) v_{A}^{x}\left(E^{\prime}\right)
\end{array}\right\} \sigma_{A}^{x}\left(E^{\prime}\right), \\
\chi_{C}^{\tau}(E) v_{C}^{\tau}(E) \sigma_{C}^{f}(E)=\sum_{A \subset C} \frac{N_{A}(\mathbf{r}, t)}{N_{C}(\mathbf{r}, t)} \chi_{A}^{\tau}(E) v_{A}^{\tau}(E) \sigma_{A}^{f}(E) .
\end{gathered}
$$

Полное макросечение $x=t$ можно записать в виде сумм

$$
\Sigma^{t}(E, \mathbf{r}, t)=\Sigma_{C}^{t}(E, \mathbf{r}, t)+M_{C}^{t}(E, \mathbf{r}, t)=N_{C}(\mathbf{r}, t) \sigma_{C}^{t}(E)+M_{C}^{t}(E, \mathbf{r}, t),
$$




$$
M_{C}^{t}(E, \mathbf{r}, t)=\sum_{D \neq C} \Sigma_{D}^{t}(E, \mathbf{r}, t)=\sum_{A \not \subset C} N_{A}(\mathbf{r}, t) \sigma_{A}^{t}(E), \quad C=C_{1}, C_{2}, \ldots
$$

где $\Sigma_{C}^{t}(E, \mathbf{r}, t)$ есть макросечение компонента «C», $M_{C}^{t}(E, \mathbf{r}, t)-$ сечение разбавления компонента.

Носители резонансов. Утверждение 1. Область резонансов можно разбить на ряд множеств $\omega_{g}=\left\{E_{p}^{-}<E<E_{p}^{+}, p=1,2, \ldots, P\right\}, 1 \leq g \leq g g$, называемых носителями резонансов. Носитель резонансов в общем случае состоит из нескольких интервалов. Относительная ширина носителя не превышает заданного числа $K_{g}$

$$
2\left[E_{P}^{+}-E_{1}^{-}\right] /\left[E_{P}^{+}+E_{1}^{-}\right]<K_{g}, \quad 1 \leq g \leq g g .
$$

(Если (15) не выполнено, то носитель резонансов всегда можно поделить на более компактные носители.) В пределах носителя резонансов полное макросечение допускает приближенное разделение переменных $E$ и $(\mathbf{r}, t)$, которое справедливо во всех точках объекта

$$
\Sigma^{t}(E, \mathbf{r}, t)=a_{g}(\mathbf{r}, t) s_{g}(E)+b_{g}(E, \mathbf{r}, t), \quad E \in \omega_{g}, \mathbf{r} \in V(t), 0 \leq t \leq T,
$$

где $a_{g}, b_{g}, s_{g} \geq 0$ - неотрицательные функции, $b_{g}(E, \mathbf{r}, t)$ есть ограниченная функция малой вариации по энергии нейтронов $E$

$$
\begin{aligned}
& \int_{\omega_{g}}\left[b_{g}(E, \mathbf{r}, t)-\bar{b}_{g}(\mathbf{r}, t)\right]^{2} d E \ll\left[a_{g}(\mathbf{r}, t) \bar{s}_{g}+\bar{b}_{g}(\mathbf{r}, t)\right]^{2} \int_{\omega_{g}} d E, \\
& \bar{b}_{g}(\mathbf{r}, t)=\int_{\omega_{g}} b_{g}(E, \mathbf{r}, t) d E / \int_{\omega_{g}} d E, \quad \bar{s}_{g}=\int_{\omega_{g}} s_{g}(E) d E / \int_{\omega_{g}} d E .
\end{aligned}
$$

Если функция не зависит от энергии $b_{g}(E, \mathbf{r}, t)=\bar{b}_{g}(\mathbf{r}, t)$, то имеет место точное разделение переменных $E$ и $(\mathbf{r}, t)$ на носителе резонансов.

Существование разбиений со свойствами (16), (17) следует из (14) и наличия резонансов. В носитель резонансов $\omega_{g}(C)$ компонента $C$ включаются интервалы энергий $\Delta E$, лежащие вблизи сильных резонансов компонента $C$ и не содержащие резонансов других компонентов. Тогда $\omega_{g}(C)$ содержит резонансы компонента $C$ и фоновое сечение (крылья резонансов) всех остальных компонентов. Формула (16) совпадает с (14) при $a_{g}=N_{C}(\mathbf{r}, t)$, $s_{g}=\sigma_{C}^{t}(E), b_{g}=M_{C}^{t}(E, \mathbf{r}, t)$, где $b_{g}$ имеет малые вариации на $\omega_{g}(C)$ в любой точке объекта $(\mathbf{r}, t)$ вне зависимости от того, содержит материал компонент $C: N_{C}(\mathbf{r}, t) \neq 0$ или не содержит $N_{C}(\mathbf{r}, t)=0$. 
Замечание. Предположение о разделении переменных $E$ и $(\mathbf{r}, t)$ во всем спектре энергий частиц $\Sigma^{t}(E, \mathbf{r}, t) \approx a_{g}(\mathbf{r}, t) s_{g}(E)(0<E<\infty)$ в теории переноса фотонов называется моделью Милна-Эддингтона.

Средние сечения. Далее термином «среднее» сечение мы будем называть полное макросечение $x=t$, осредненное по объему объекта и характерному интервалу времени, если не оговорено иное. Среднее полное макросечение $S^{t}(E)$ [см $\left.{ }^{-1}\right]$ есть сумма средних полных макросечений компонентов $S_{C}^{t}(E)$. Средние зависят только от энергии нейтрона $E$

$$
\begin{aligned}
& S^{t}(E)=\left\langle\Sigma^{t}\right\rangle=\frac{1}{T} \int_{0}^{T} \frac{d t}{V(t)} \int_{V(t)} K(\mathbf{r}, t) \Sigma^{t}(E, \mathbf{r}, t) d \mathbf{r}=\sum_{C} S_{C}^{t}(E), \\
& S_{C}^{t}(E)=\frac{1}{T} \int_{0}^{T} \frac{d t}{V(t)} \int_{V(t)} K(\mathbf{r}, t) N_{C}(\mathbf{r}, t) \sigma_{C}^{t}(E) d \mathbf{r}, \quad \frac{1}{T} \int_{0}^{T} \frac{d t}{V(t)} \int_{V(t)} K(\mathbf{r}, t) d \mathbf{r}=1 .
\end{aligned}
$$

$K(\mathbf{r}, t)$ - весовая функция, выделяющая сечения в областях объекта, если того требует специфика задачи. В неспецифичном случае $K(\mathbf{r}, t)=1$.

Алгоритм разбиения области резонансов на носители использует средние сечения (18).

А) Интервал энергий $\Delta E$ включается в носитель резонансов $\omega_{g}(C)$, в который собираются резонансы компонента $C$, если на интервале среднее макросечение компонента больше среднего макросечения любого другого резонансного компонента $S_{C}^{t}(E)>S_{D}^{t}(E), D \neq C$ (рис.2).

Б) Если на интервале $\Delta E$ преобладает среднее сечение компонента, не имеющего резонансов, например - замедлителя нейтронов, то интервал $\Delta E$ включается в ближайший носитель. Компоненты, представленные в объекте в малых концентрациях, могут не получить носителей своих резонансов.

В) Если какой-либо носитель содержит «широкие» резонансы, ширина которых превышает ширину индикатрисы рассеяния $\Delta\left(E^{\prime}\right)$ (см. (10)), то он разбивается на два: носитель левых крыльев резонансов, состоящий из интервалов роста сечения $S^{t}(E)$, и носитель правых крыльев, состоящий из интервалов уменьшения сечения. Это позволит учесть асимметрию спектра замедляющихся нейтронов, возникающую в крыльях широких резонансов.

Пример. Если объект состоит из зон, заполненных компонентом-замедлителем, не имеющим резонансов, и резонансным компонентом, имеющим «узкие» резонансы, то в соответствие с алгоритмом область резонансов делится на обычные группы $\omega_{g}=\left\{E_{g-1}<E<E_{g}\right\}$. Число групп $g g=I$ зави- 
сит от выбора числа $K_{g}$ в (15). Если объект состоит из зон, заполненных замедлителем и резонансными компонентами $C$ и $D$, то область резонансов разбивается на носители резонансов компонента $C$ и компонента $D$ (рис. 2). Общее число носителей $g g$ не превысит $2 I$.

Утвержжение 2. На носителе резонансов локальное макросечение любой группы реакций «x» в точке $(\mathbf{r}, t)$ связано со средним по объекту полным макросечением $S^{t}(E)$ зависимостью, близкой к линейной

$$
\Sigma^{x}(E, \mathbf{r}, t)=A_{g}^{x}(\mathbf{r}, t) S^{t}(E)+B_{g}^{x}(E, \mathbf{r}, t), \quad E \in \omega_{g}, \mathbf{r} \in V(t), 0 \leq t \leq T,
$$

где $B_{g}^{x}(E, \mathbf{r}, t)$ есть функция малой вариации по аргументу $E$ в любой точке объекта. Если в некоторых зонах объекта функция $A_{g}^{x}(\mathbf{r}, t)$ равна нулю, то макросечение определяется только функцией $B_{g}^{x}$.

Для доказательства утверждения в случае $x=t$ осредним (16) по объекту, выразим $s_{g}(E)$ через $S^{t}(E)$ и подставим в (16). Мы получим (19) с коэффициентами $A_{g}^{t}(\mathbf{r}, t)=a_{g}(\mathbf{r}, t) /\left\langle a_{g}\right\rangle, B_{g}^{t}(E, \mathbf{r}, t)=b_{g}(E, \mathbf{r}, t)-A_{g}^{t}(\mathbf{r}, t)\left\langle b_{g}\right\rangle$. Из формул Брейта-Вигнера (8) следует, что микросечение группы реакций «x» и полное микросечение связаны зависимостью, близкой к линейной $\sigma_{A}^{x}(E)=d_{A}^{x} \sigma_{A}^{t}(E)+e_{A}^{x}$. Здесь $d_{A}^{x} \sim \Gamma^{x} / \Gamma$ и $e_{A}^{x}$ есть медленно меняющиеся функции энергии нейтрона в сравнении с $\sigma_{A}^{t}(E)$. Поэтому (19) распространяется на любую группу реакций «x». Далее индекс «t» для среднего полного макросечения иногда будем опускать: $S(E) \equiv S^{t}(E)$.

Утвержљение 3. В формировании поля нейтронов участвуют все материалы и зоны объекта. На носителе резонансов в любой точке объекта $(\mathbf{r}, t)$ ток нейтронов можно представить в виде функции

$$
\varphi(E, \boldsymbol{\Omega}, \mathbf{r}, t)=F(E) \cdot\left[Z_{g}(S(E), \boldsymbol{\Omega}, \mathbf{r}, t)+\varepsilon_{g}(E, \boldsymbol{\Omega}, \mathbf{r}, t)\right], \quad E \in \omega_{g},
$$

где $\varepsilon_{g}(E)$ и $F(E)$ - функции малой вариации по $E, F(E)$ - характерный для задачи синтетический спектр (см. (11)), $Z_{g}>0$ есть сложная функция, зависящая от энергии нейтронов через среднее по объекту сечение $S(E)$.

Рассмотрим стационарную задачу без отражения частиц от внешних границ. В более общем случае доказательство проводится аналогично. Запишем решение уравнения переноса (1) вдоль характеристики в пределах зоны объекта, содержащей однородный материал 


$$
\begin{aligned}
\varphi(E, l) & =\int_{l_{\text {in }}}^{l} q(\varphi) \exp \left[-\int_{l^{\prime}}^{l} \Sigma^{t}\left(E, l^{\prime \prime}\right) d l^{\prime \prime}\right] d l^{\prime}+\varphi\left(E, l_{\text {in }}\right) \exp \left[-\int_{l_{\text {in }}}^{l} \Sigma^{t}\left(E, l^{\prime}\right) d l^{\prime}\right] \approx \\
& \approx \frac{q(\varphi)}{\Sigma^{t}(E, l)}\left[1-\exp \left[-\int_{l_{\text {in }}}^{l} \Sigma^{t}\left(E, l^{\prime}\right) d l^{\prime}\right]\right]+\varphi\left(E, l_{\text {in }}\right) \exp \left[-\int_{l_{\text {in }}}^{l} \Sigma^{t}\left(E, l^{\prime}\right) d l^{\prime}\right] .
\end{aligned}
$$

Здесь $l$ и $l_{\text {in }}$ - характеристические координаты точки наблюдения $\mathbf{r}$ и точки входа характеристики в зону $\mathbf{r}_{i n}=\mathbf{r}-\boldsymbol{\Omega}\left[l-l_{i n}\right], \varphi\left(E, l_{i n}\right)$ - ток нейтронов на границе зоны. Приближенная оценка тока нейтронов справедлива, т.к. в пределах зоны сечение $\Sigma^{t}(E, l)$ меняется непрерывно. Формула (21) описывает выход решения на асимптотику стационарного режима (11), который устанавливается в глубине зоны бесконечной протяженности. Скорость сближения с асимптотикой зависит от величины сечения $\Sigma^{t}(E, l)$ при разных энергиях нейтронов. Подставим в (21) представление сечения на носителе резонансов в виде (19). Тогда (21) приводится к (20), если к (20) приводится ток нейтронов на границе зоны $\varphi\left(E, l_{i n}\right)$. Будем двигаться вдоль характеристики от точки входа в объект, где это так. Последовательно вычисляя функции $\varphi\left(E, l_{\text {in }}\right)$ в точках пересечения границ между зонами объекта, мы всякий раз имеем возможность приводить решение (21) к виду (20).

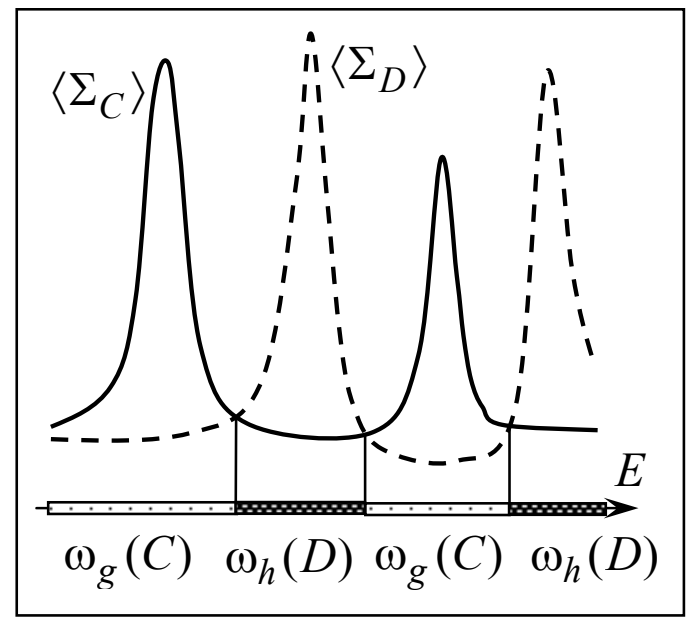

Рис.2. Разбиение спектра на носители резонансов компонентов C и D.

\section{2. Лебегово осреднение}

В разделе изложено осреднение сечений и тока нейтронов по системе лебеговых множеств. Множества строятся независимо в каждом носителе резонансов. От читателя не требуется знаний теории меры и интеграла Ле- 
бега. Мы воспользуемся лишь формальной конструкцией интеграла, которая подходит для осреднения резонансных функций. Все функции ограничены, интегрируемы по Риману и Лебегу, и оба интеграла равны.

Система вложенных множеств. Построим внутри носителя $g$ систему множеств (рис.3), включив в множество $\omega(g, S)$ такие энергии $E$, при которых среднее сечение (18) не превышает уровня $S, S(E)<S$. Множество состоит из серии интервалов $E_{k}^{-}(S)<E<E_{k}^{+}(S), k=1,2, \ldots$. Левые и правые границы интервалов $E_{k}^{ \pm}(S)$ - это точки с одинаковым средним сечением $S=S\left(E_{k}^{ \pm}\right)$или границы носителя резонансов. При увеличении уровня $S$ левые границы могут смещаться только влево, правые границы - только вправо. Поэтому имеет место вложение множеств: $\omega\left(S_{0 g}\right) \subseteq \omega(S) \subseteq \omega\left(S_{g}\right)$, $S_{0 g} \leq S \leq S_{g} \cdot S_{0 g}$ - уровень, при котором множество пусто $\omega\left(S_{0 g}\right)=\varnothing, S_{g}$ - уровень, при котором множество совпадает с носителем резонансов $\omega_{g}$.

Введем важную для дальнейших построений величину - меру $m(S)$ [эВ] лебегова множества $\omega(g, S)$, определяемую с помощью интеграла

$$
\begin{gathered}
m(S)=\int_{\omega(g, S)} F(E) d E=\sum_{k} \int_{E_{k}^{-}(S)}^{E_{k}^{+}(S)} F(E) d E, \quad \begin{array}{l}
0 \leq m(S) \leq m_{g} \\
m_{0 g} \leq S \leq S_{g},
\end{array} \\
m_{g}=m\left(S_{g}\right)=0, \quad \int_{\omega_{g}} F(E) d E
\end{gathered}
$$

где $F(E)$ - безразмерный весовой спектр, пропорциональный, например, спектру Вигнера (см. (11)). Если спектр указать затруднительно, то полагаем $F=1$. Спектр можно уточнять при решении типовых задач. Мера меняется от нуля до интеграла от весового спектра по носителю резонансов. В случае $F=1$ мера равна сумме длин интервалов, на которых $S(E)<S$.

Замечание. Лебегово осреднение можно проводить с опорными функциями $S(E, \mathbf{r}, t), F(E, \mathbf{r}, t)$, зависящими от координат [7-10]. В задачах переноса нейтронов достаточно рассматривать случай $S=S(E), F=F(E)$.

Так как мера есть неубывающая функция уровня $S$, то существует неубывающая обратная функция, задающая зависимость уровня от меры $S=S(m)\left(S_{0 g} \leq S(m) \leq S_{g}\right)$. Параметризуем систему множеств $\omega(g, S)$ через их меру $m$, выполнив с помощью обратной функции замену переменных $\omega(g, S(m))=\omega(g, m)$. При $m=0$ множество пусто $\omega(0)=\varnothing$. При $m=m_{g}$ множество $\omega\left(m_{g}\right)$ плотно заполняет носитель резонансов $\omega_{g}$. Часть гранич- 
ных точек множества $E_{k}^{ \pm}(S(m))=E_{k}^{ \pm}(m)$ - это точки с одинаковым средним сечением $S\left(E_{k}^{ \pm}\right)=S(m)$. В них $\partial E_{k}^{+} / \partial m \geq 0, \partial E_{k}^{-} / \partial m \leq 0$. Остальные точки это границы носителя резонансов: $\partial E_{k}^{ \pm} / \partial m=0$.

Дифференцируя (22) по мере $m$ при условии, что она выбрана в качестве независимой переменной, получим тождество, выражающее взаимосвязь производных прямой и обратной функций

$$
\frac{\partial m}{\partial m}=\frac{\partial}{\partial m} \sum_{k} \int_{E_{k}^{-}(m)}^{E_{k}^{+}(m)} F(E) d E=\sum_{k}\left[F\left(E_{k}\right) \frac{\partial E_{k}}{\partial m}\right]_{-}^{+}=1,
$$

где $\left[F\left(E_{k}\right) \frac{\partial E_{k}}{\partial m}\right]_{-}^{+}=F\left(E_{k}^{+}\right) \frac{\partial E_{k}^{+}}{\partial m}-F\left(E_{k}^{-}\right) \frac{\partial E_{k}^{-}}{\partial m}, \quad \frac{\partial E_{k}^{+}}{\partial m} \geq 0, \quad \frac{\partial E_{k}^{-}}{\partial m} \leq 0$.

Суммирование выполняется по граничным точкам $E_{k}^{ \pm}$с одинаковым средним сечением $S\left(E_{k}^{ \pm}\right)=S(m)$. Границы носителя резонансов из суммы выпадают, т.к. в них $\partial E_{k}^{ \pm} / \partial m=0$. Все слагаемые суммы неотрицательны.

Лебегов ток нейтронов. Пусть $T_{g}(m, \boldsymbol{\Omega}, \mathbf{r}, t)\left[\mathrm{cm}^{-2} \mathrm{ce \kappa}^{-1}\right]$ есть кумулятивное (нарастающим итогом) распределение нейтронов на системе вложенных множеств, построенной внутри носителя резонансов $g$ :

$$
T_{g}(m, \mathbf{\Omega}, \mathbf{r}, t)=\int_{\omega(g, m)} \varphi(E, \mathbf{\Omega}, \mathbf{r}, t) d E=\sum_{k} \int_{E_{k}^{-}(m)}^{E_{k}^{+}(m)} \varphi(E, \mathbf{\Omega}, \mathbf{r}, t) d E .
$$

При $m=0$ распределение равно нулю, при $m=m_{g}$ оно равно интегралу от тока нейтронов $\varphi(E)$ по носителю резонансов $g$. Лебеговым током нейтронов $\psi_{g}(m, \boldsymbol{\Omega}, \mathbf{r}, t)\left[{ }^{-1} \mathrm{~B}^{-1} \mathrm{~cm}^{-2}\right.$ сек $\left.^{-1}\right]$ будем называть плотность распределения

$$
\begin{gathered}
\psi_{g}(m, \boldsymbol{\Omega}, \mathbf{r}, t)=\frac{\partial T_{g}}{\partial m}=\frac{\partial}{\partial m} \sum_{k} \int_{E_{k}^{-}(m)}^{E_{k}^{+}(m)} \varphi(E, \boldsymbol{\Omega}, \mathbf{r}, t) d E=\sum_{k}\left[\varphi\left(E_{k}\right) \frac{\partial E_{k}}{\partial m}\right]_{-}^{+}= \\
=\sum_{k}\left[\frac{\varphi\left(E_{k}\right)}{F\left(E_{k}\right)} F\left(E_{k}\right) \frac{\partial E_{k}}{\partial m}\right]_{-}^{+}=\left\langle\frac{\varphi\left(E_{k}^{ \pm}\right)}{F\left(E_{k}^{ \pm}\right)}\right\rangle \cdot \sum_{k}\left[F\left(E_{k}\right) \frac{\partial E_{k}}{\partial m}\right]_{-}^{+}=\left\langle\frac{\varphi\left(E_{k}^{ \pm}\right)}{F\left(E_{k}^{ \pm}\right)}\right\rangle .
\end{gathered}
$$

Последнее равенство получено с учетом тождества (23). Как видим, лебегов ток $\psi_{g}(m, \mathbf{\Omega}, \mathbf{r}, t)$ есть среднее отношение тока нейтронов $\varphi(E, \boldsymbol{\Omega}, \mathbf{r}, t)$ к весовому спектру $F(E)$, вычисляемое по совокупности граничных точек ле- 
бегова множества $E_{k}^{ \pm}=E_{k}^{ \pm}(m), k=1,2, \ldots$, в которых среднее макросечение принимает одно и то же значение $S\left(E_{k}^{ \pm}\right)=S(m)$. Определение корректно, поскольку все слагаемые суммы неотрицательны. Зависимость лебегова тока $\psi_{g}(m)$ от меры $m$ соответствует зависимости тока нейтронов $\varphi(E)$ от энергии $E$. Суммирование по точкам $E_{k}^{ \pm}(m)$ с весом $\left|\partial E_{k}^{ \pm} / \partial m\right|$ не уменьшает число независимых аргументов функций.

Пример. Пусть лебегов ток не зависит от $m$ :

$$
T_{g}(m, \boldsymbol{\Omega}, \mathbf{r}, t)=\int_{0}^{m} \frac{\partial T_{g}}{\partial m} d m=\int_{0}^{m} \psi_{g}(m, \mathbf{\Omega}, \mathbf{r}, t) d m=\psi_{g}(\boldsymbol{\Omega}, \mathbf{r}, t) \cdot m .
$$

Полагая $m=m_{g}$, мы найдем: $\psi_{g}(\boldsymbol{\Omega}, \mathbf{r}, t)=T_{g}\left(m_{g}, \boldsymbol{\Omega}, \mathbf{r}, t\right) / m_{g}$. Т.е. лебегов ток равен отношению интеграла от тока нейтронов на носителе резонансов $\omega_{g}$ к соответствующему интегралу от весового спектра.

Вычисление интегралов. Важным прикладным свойством интеграла Лебега является возможность экономно вычислять интегралы от немонотонных, резонансных функций многих переменных

$$
I(m, \boldsymbol{\Omega}, \mathbf{r}, t)=\int_{\omega(g, m)} Y(E, \mathbf{r}, t) \varphi(E, \boldsymbol{\Omega}, \mathbf{r}, t) d E, \quad 0<m \leq m_{g},
$$

где $Y(E, \mathbf{r}, t)=X(S(E), \mathbf{r}, t)$ - некоторая заданная сложная функция, зависящая от энергии $E$ через относительно «хорошую» функцию $X(S)$ и немонотонную функцию $S(E) ; \varphi$ - ток нейтронов. При $m=m_{g}$ интегрирование выполняется по носителю резонансов $g$. Численный расчет интегралов (26) по Риману в точках пространства $E \times \mathbf{\Omega} \times \mathbf{r} \times t$ приводит к большому объему вычислений на подробной неравномерной сетке. Точность вычислений зависит от искусства подбора сетки. С другой стороны, если на основе функции $S(E)$ построить систему лебеговых множеств, то интегралы можно представить в виде

$$
\begin{aligned}
I=\int_{0}^{m} & \frac{\partial}{\partial m}\left[\int_{\omega(g, m)} Y(E) \varphi(E) d E\right] d m=\int_{0}^{m} \sum_{k}\left[X\left(S\left(E_{k}\right)\right) \varphi\left(E_{k}\right) \frac{\partial E_{k}}{\partial m}\right]_{-}^{+} d m= \\
& =\int_{0}^{m} X(S(m)) \sum_{k}\left[\varphi\left(E_{k}\right) \frac{\partial E_{k}}{\partial m}\right]_{-}^{+} d m=\int_{0}^{m} X(S(m), \mathbf{r}, t) \psi_{g}(m, \mathbf{\Omega}, \mathbf{r}, t) d m .
\end{aligned}
$$

Функция $X\left(S\left(E_{k}^{ \pm}\right)\right)$вынесена из суммы, т.к. она принимает одинаковые значения $X(S(m))$ в точках $E_{k}^{ \pm}$. Уровень $S(m)$ есть неубывающая функция $m$ вне зависимости от того - один или тысячу резонансов имеет функция 
$S(E)$ на носителе $\omega_{g}$. Функции $X(S(m))$ и $\psi_{g}(m)$ имеют хорошие свойства монотонности по аргументу $m$. Поэтому вычисление интегралов можно проводить на редкой сетке с малыми затратами ресурсов.

Выигрыш от перехода к лебеговым распределениям достигается за счет объединения точек с одинаковым макросечением $S\left(E_{k}^{ \pm}\right)=S(m)$ в одну расчетную точку и монотонизации сечений. Величина выигрыша примерно равна числу резонансов на носителе. Функции $X(S), S(E)$ должны быть известны заранее. Соотношение между вычислением интегралов (26), (27) от немонотонных функций по Риману и по Лебегу такое же, как между использованием непозиционной и позиционной системы счисления - «яйца можно считать поштучно, а можно - десятками».

Вычислим скорость ухода нейтронов из пучков, энергия которых лежит в пределах множества $E \in \omega(g, m) \subseteq \omega_{g}$, и скорости испускания нейтронов, осколков деления (см. (2)) и нуклидов (см. (7)) в реакциях «хх $\left\{\begin{array}{c}I_{g}^{x}(m, \boldsymbol{\Omega}, \mathbf{r}, t) \\ J_{g}^{x}(m, \boldsymbol{\Omega}, \mathbf{r}, t)\end{array}\right\}=\int_{\omega(g, m)}\left\{\begin{array}{c}1 \\ v^{x}(E, \mathbf{r}, t)\end{array}\right\} \times \Sigma^{x}(E, \mathbf{r}, t) \varphi(E, \boldsymbol{\Omega}, \mathbf{r}, t) d E, \quad 0<m \leq m_{g}$. Представим интегралы в форме интегралов Лебега (27)

$$
\begin{aligned}
& \left\{\begin{array}{c}
I_{g}^{x} \\
J_{g}^{x}
\end{array}\right\}=\int_{0}^{m} \frac{\partial}{\partial m}\left[\int_{\omega(g, m)}\left\{\begin{array}{c}
1 \\
v^{x}(E, \mathbf{r}, t)
\end{array}\right\} \times \Sigma^{x}(E, \mathbf{r}, t) \varphi(E, \mathbf{\Omega}, \mathbf{r}, t) d E\right] d m= \\
& =\int_{0}^{m} \sum_{k}\left[\left\{\begin{array}{c}
1 \\
v^{x}
\end{array}\right\} \Sigma^{x} F \frac{\varphi}{F} \frac{\partial E_{k}}{\partial m}\right]_{-}^{+} d m \approx \int_{0}^{m} \psi_{g}(m) \sum_{k}\left[\left\{\begin{array}{c}
1 \\
v^{x}\left(E_{k}\right)
\end{array}\right\} \Sigma^{x}\left(E_{k}\right) F\left(E_{k}\right) \frac{\partial E_{k}}{\partial m}\right]_{-}^{+} d m \approx \\
& \approx \int_{0}^{m}\left\{\begin{array}{c}
1 \\
v^{x}(m, \mathbf{r}, t)
\end{array}\right\} \times \Sigma_{g}^{x}(m, \mathbf{r}, t) \psi_{g}(m, \mathbf{\Omega}, \mathbf{r}, t) d m \text {. }
\end{aligned}
$$

Здесь отношения $\varphi\left(E_{k}^{ \pm}\right) / F\left(E_{k}^{ \pm}\right)$в граничных точках $E_{k}^{ \pm}$заменены своим средним $\left\langle\varphi\left(E_{k}^{ \pm}\right) / F\left(E_{k}^{ \pm}\right)\right\rangle=\psi_{g}(m)$, вычисляемым по этим же точкам. Функции $\Sigma_{g}^{x}(m, \mathbf{r}, t)$ и $v^{x}(m, \mathbf{r}, t)$ называются соответственно лебеговым сечением и лебеговой кратностью выхода частиц в группе реакций «х»:

$$
\begin{gathered}
\left\{1, v_{g}^{x}(m, \mathbf{r}, t)\right\} \times \Sigma_{g}^{x}(m, \mathbf{r}, t)=\frac{\partial}{\partial m} \int_{\omega(m)}\left\{1, v^{x}(E, \mathbf{r}, t)\right\} \times \Sigma^{x}(E, \mathbf{r}, t) F(E) d E= \\
=\sum_{k}\left[\left\{1, v^{x}\left(E_{k}, \mathbf{r}, t\right)\right\} \times \Sigma^{x}\left(E_{k}, \mathbf{r}, t\right) F\left(E_{k}\right) \partial E_{k} / \partial m\right]_{-}^{+} .
\end{gathered}
$$


Лебегово сечение есть среднее сечение, вычисляемое по совокупности граничных точек $E_{k}^{ \pm}, S\left(E_{k}^{ \pm}\right)=S(m)$ с весом $F\left(E_{k}^{ \pm}\right) \times\left|\partial E_{k}^{ \pm} / \partial m\right|$.

В отличие от (27), формула (28) дает приближенные значения интегралов. Оценим допущенную ошибку:

$$
\begin{gathered}
\Delta(m, \boldsymbol{\Omega}, \mathbf{r}, t)=\int_{0}^{m} \delta(m, \mathbf{\Omega}, \mathbf{r}, t) d m \\
\delta(m, \mathbf{\Omega}, \mathbf{r}, t)=\sum_{k}\left[\left[\Sigma^{x}\left(E_{k}\right)-\Sigma_{g}^{x}(m)\right]\left[\frac{\varphi\left(E_{k}\right)}{F\left(E_{k}\right)}-\psi_{g}(m)\right] F\left(E_{k}\right) \frac{\partial E_{k}}{\partial m}\right]_{-}^{+}= \\
=\sum_{k}\left[\left[B_{g}^{x}\left(E_{k}, \mathbf{r}, t\right)-B_{g}^{x}(m, \mathbf{r}, t)\right]\left[\varepsilon_{g}\left(E_{k}, \boldsymbol{\Omega}, \mathbf{r}, t\right)-\varepsilon_{g}(m, \boldsymbol{\Omega}, \mathbf{r}, t)\right] F\left(E_{k}\right) \frac{\partial E_{k}}{\partial m}\right]_{-}^{+} \\
\left\{\begin{array}{c}
B_{g}^{x}(m, \mathbf{r}, t) \\
\varepsilon_{g}(m, \mathbf{\Omega}, \mathbf{r}, t)
\end{array}\right\}=\sum_{k}\left[\left\{\begin{array}{c}
B_{g}^{x}\left(E_{k}\right) \\
\varepsilon_{g}\left(E_{k}\right)
\end{array}\right\} F\left(E_{k}\right) \frac{\partial E_{k}}{\partial m}\right]_{-}^{+}=\frac{\partial}{\partial m} \int_{\omega(m)}\left\{\begin{array}{c}
B_{g}^{x}(E, \mathbf{r}, t) \\
\varepsilon_{g}(E, \boldsymbol{\Omega}, \mathbf{r}, t)
\end{array}\right\} F(E) d E .
\end{gathered}
$$

Здесь были использованы представления сечения (19) и тока нейтронов (20) на носителе резонансов $g$ в форме разделенных переменных $E$ и $(\mathbf{r}, t)$. Слагаемые $A_{g}^{x}(\mathbf{r}, t) S\left(E_{k}^{ \pm}\right), Z_{g}\left(S\left(E_{k}^{ \pm}\right), \boldsymbol{\Omega}, \mathbf{r}, t\right)$ из суммы выпадают, т.к. они принимают одинаковые значения $A_{g}^{x}(\mathbf{r}, t) S(m), Z_{g}(S(m), \boldsymbol{\Omega}, \mathbf{r}, t)$ в точках суммирования $E_{k}^{ \pm}$. Ошибка зависит только от функций малой вариации $B_{g}^{x}\left(E_{k}^{ \pm}\right)$и $\varepsilon_{g}\left(E_{k}^{ \pm}\right)$. Величины $B_{g}^{x}(m)$ и $\varepsilon_{g}(m)$ есть средние значения функций, вычисляемые по точкам суммирования.

Для малости ошибки $\delta$ достаточно выполнения одного из неравенств:

$$
\begin{gathered}
\left\{\begin{array}{c}
D(m, B) \\
D(m, \varepsilon)
\end{array}\right\}=\sum_{k}\left[\left\{\begin{array}{c}
{\left[B_{g}^{x}\left(E_{k}\right)-B_{g}^{x}(m)\right]^{2}} \\
{\left[\varepsilon_{g}\left(E_{k}\right)-\varepsilon_{g}(m)\right]^{2}}
\end{array}\right\} F\left(E_{k}\right) \frac{\partial E_{k}}{\partial m}\right]_{-}^{+} \ll\left\{\begin{array}{l}
{\left[\Sigma_{g}^{x}(m, \mathbf{r}, t)\right]^{2}} \\
\psi_{g}^{2}(m, \boldsymbol{\Omega}, \mathbf{r}, t)
\end{array}\right\}, \\
\left\{\begin{array}{c}
\Sigma_{g}^{x}(m, \mathbf{r}, t) \\
\psi_{g}(m, \boldsymbol{\Omega}, \mathbf{r}, t)
\end{array}\right\}=\left\{\begin{array}{c}
A_{g}^{x}(\mathbf{r}, t) S(m)+B_{g}^{x}(m, \mathbf{r}, t) \\
Z_{g}(S(m), \boldsymbol{\Omega}, \mathbf{r}, t)+\varepsilon_{g}(m, \boldsymbol{\Omega}, \mathbf{r}, t)
\end{array}\right\} .
\end{gathered}
$$

Здесь $D(m, B), D(m, \varepsilon)$ - дисперсии распределений $B_{g}^{x}\left(E_{k}^{ \pm}\right), \varepsilon_{g}\left(E_{k}^{ \pm}\right)$на совокупности граничных точек $E_{k}^{ \pm}, k=1,2, \ldots$ Так как $B_{g}^{x}(E), \varepsilon_{g}(E)-$ функции малой вариации (следствие разделения переменных на носителе резонансов), то оба неравенства (31) выполняются одновременно. Ошибка в вычислении скоростей (28) пренебрежимо мала. 
Также ошибка $\delta(m, \mathbf{\Omega}, \mathbf{r}, t)$ равна нулю, если суммы по «k» содержат только одно слагаемое. Это происходит в «коридорах» монотонности сечения $S_{g l}<S^{t}(E)<S_{g l+1}$. Если уменьшать ширину носителей резонансов (число $K_{g}$ в (15)), то наступит момент, когда коридоры сольются в один коридор, среднее сечение $S^{t}(E)$ станет монотонной функцией на всем носителе резонансов, и полная ошибка $\Delta(m, \boldsymbol{\Omega}, \mathbf{r}, t)$ обратится в ноль.

Скорости прихода нейтронов $Q_{g}^{x}(m, \boldsymbol{\Omega}, \mathbf{r}, t)$ и $q_{g}^{x}(\psi)=\partial Q_{g}^{x}(m) / \partial m$ в множество $E \in \omega(g, m)$ и единичный интервал меры «m» рассчитываются в соответствии с общим правилом «осреднения по начальным состояниям переходов и суммирования по конечным состояниям». Вначале выполним лебегово суммирование по точкам носителя резонансов $E^{\prime} \in \omega\left(h, m^{\prime}\right) \subseteq \omega_{h}$, с которого уходят первичные нейтроны; $m^{\prime}\left(0 \leq m^{\prime} \leq m_{h}\right)$ - мера вложенных в него множеств. Зависимость величин от $(\mathbf{r}, t)$ временно опустим:

$$
\begin{aligned}
& Q_{g}^{x}(m, \mathbf{\Omega})=\int_{\omega(g, m)} d E \int_{4 \pi} \frac{d \mathbf{\Omega}^{\prime}}{2 \pi} \int_{0}^{\infty} v^{x}\left(E^{\prime}\right) \Sigma^{x}\left(E^{\prime}\right) W^{x}\left(E^{\prime} \rightarrow E, \eta\right) \varphi\left(E^{\prime}, \mathbf{\Omega}^{\prime}\right) d E^{\prime}=\text { (32) } \\
& =\int_{\omega(g, m)} d E \int_{4 \pi} \frac{d \mathbf{\Omega}^{\prime}}{2 \pi} \sum_{h} \int_{0}^{m_{h}} \sum_{k}\left[v^{x}\left(E_{k}^{\prime}\right) \Sigma^{x}\left(E_{k}^{\prime}\right) W^{x}\left(E_{k}^{\prime} \rightarrow E, \eta\right) \varphi\left(E_{k}^{\prime}, \mathbf{\Omega}^{\prime}\right) \frac{\partial E_{k}^{\prime}}{\partial m^{\prime}}\right]_{-}^{+} d m^{\prime} \approx \\
& \approx \int_{\omega(g, m)} d E \int_{4 \pi} \frac{d \mathbf{\Omega}^{\prime}}{2 \pi} \sum_{h} \int_{0}^{m_{h}} v_{h}^{x}\left(m^{\prime}\right) \Sigma_{h}^{x}\left(m^{\prime}\right) W_{h}^{x}\left(m^{\prime} \rightarrow E, \eta\right) \psi_{h}\left(m^{\prime}, \mathbf{\Omega}^{\prime}\right) d m^{\prime}=\int_{0}^{m} q_{g}^{x}(\psi) d m, \\
& v_{h}^{x}\left(m^{\prime}\right) \Sigma_{h}^{x}\left(m^{\prime}\right) W_{h}^{x}\left(m^{\prime} \rightarrow E, \eta\right)=\frac{\partial}{\partial m^{\prime}} \int_{\omega\left(h, m^{\prime}\right)} v^{x}\left(E^{\prime}\right) \Sigma^{x}\left(E^{\prime}\right) W^{x}\left(E^{\prime} \rightarrow E, \eta\right) F\left(E^{\prime}\right) d E^{\prime} .
\end{aligned}
$$

На втором этапе выполним суммирование по граничным точкам множества $E \in \omega(g, m)$, в которое приходят мгновенные нейтроны:

$$
\begin{aligned}
& q_{g}^{x}(\psi)=\frac{\partial}{\partial m} \int_{\omega(g, m)} d E \int_{4 \pi} \frac{d \mathbf{\Omega}^{\prime}}{2 \pi} \sum_{h} \int_{0}^{m_{h}} v_{h}^{x}\left(m^{\prime}\right) \Sigma_{h}^{x}\left(m^{\prime}\right) W_{h}^{x}\left(m^{\prime} \rightarrow E, \eta\right) \psi_{h}\left(m^{\prime}, \mathbf{\Omega}^{\prime}\right) d m^{\prime}=(33) \\
& =\int_{4 \pi} \frac{d \mathbf{\Omega}^{\prime}}{2 \pi} \sum_{h} \int_{0}^{m_{h}} v_{h}^{x}\left(m^{\prime}, \mathbf{r}, t\right) \sum_{h}^{x}\left(m^{\prime}, \mathbf{r}, t\right) W_{h \rightarrow g}^{x}\left(m^{\prime} \rightarrow m, \eta, \mathbf{r}, t\right) \psi_{h}\left(m^{\prime}, \mathbf{\Omega}^{\prime}, \mathbf{r}, t\right) d m^{\prime} .
\end{aligned}
$$

Индикатриса реакций $W_{h \rightarrow g}^{x}\left(m^{\prime} \rightarrow m, \eta, \mathbf{r}, t\right)$ определяется формулой 


$$
\begin{aligned}
& v_{h}^{x}\left(m^{\prime}, \mathbf{r}, t\right) \Sigma_{h}^{x}\left(m^{\prime}, \mathbf{r}, t\right) W_{h \rightarrow g}^{x}\left(m^{\prime} \rightarrow m, \eta, \mathbf{r}, t\right)= \\
& =\frac{\partial^{2}}{\partial m \partial m^{\prime}} \int_{\omega(g, m)} d E \int_{\omega\left(h, m^{\prime}\right)} v^{x}\left(E^{\prime}, \mathbf{r}, t\right) \Sigma^{x}\left(E^{\prime}, \mathbf{r}, t\right) W^{x}\left(E^{\prime} \rightarrow E, \eta, \mathbf{r}, t\right) F\left(E^{\prime}\right) d E^{\prime} .
\end{aligned}
$$

Она описывает переходы нейтронов вверх и вниз по переменной спектра $m$ в пределах носителя резонансов $h=g$, переходы нейтронов между разными носителями $h \neq g$ и удовлетворяет условию нормировки (сравни с (6))

$$
\sum_{g} \int_{0}^{m_{g}} d m \int_{-1}^{1} W_{h \rightarrow g}^{x}\left(m^{\prime} \rightarrow m, \eta, \mathbf{r}, t\right) d \eta=1, \quad \eta=\mathbf{\Omega} \mathbf{\Omega}^{\prime}
$$

Ошибка в вычислении скорости прихода нейтронов (33) есть сумма пренебрежимо малых ошибок вычисления скоростей ухода нейтронов (28).

Уравнение переноса для лебегова тока нейтронов $\psi_{g}(m, \boldsymbol{\Omega}, \mathbf{r}, t)$. Интегрирование уравнения (1) по системе вложенных множеств $E \in \omega(g, m)$ дает уравнение для кумулятивного распределения (24). Взяв производную по мере $m$, мы получим искомое уравнение

$$
\left[\frac{1}{v_{g}} \frac{\partial}{\partial t}+\Omega_{i} \frac{\partial}{\partial r_{i}}+\Sigma_{g}^{t}(m, \mathbf{r}, t)\right] \psi_{g}(m, \boldsymbol{\Omega}, \mathbf{r}, t)=q_{g}^{s}(\psi)+q_{g}^{e x t}(\psi) .
$$

Лебеговы сечения $\Sigma_{g}^{x}, v_{g}^{x}$ и источник мгновенных нейтронов $q_{g}^{s}(\psi)$ даются формулами (29), (33), (34). Источник $q_{g}^{e x t}(\psi)$ и скорость $v_{g}-$ формулами

$$
\begin{aligned}
& q_{g}^{\text {ext }}(\psi)=\sum_{\tau} \frac{\chi_{g}^{\tau}(m, \mathbf{r}, t)}{4 \pi} \lambda^{\tau} P^{\tau}(\mathbf{r}, t)+q_{g}^{r}(m, \mathbf{\Omega}, \mathbf{r}, t), \quad \frac{1}{v_{g}}=\frac{1}{m_{g}} \int_{\omega_{g}} \frac{F(E)}{v} d E, \\
& \left\{\begin{array}{c}
\chi_{g}^{\tau}(m, \mathbf{r}, t) \\
q_{g}^{r}(m, \boldsymbol{\Omega}, \mathbf{r}, t)
\end{array}\right\}=\frac{\partial}{\partial m} \int_{\omega(g, m)}\left\{\begin{array}{c}
\chi^{\tau}(E, \mathbf{r}, t) \\
q^{r}(E, \boldsymbol{\Omega}, \mathbf{r}, t)
\end{array}\right\} d E, \quad \sum_{g} \int_{0}^{m_{g}} \chi_{g}^{\tau}(m, \mathbf{r}, t) d m=1 .
\end{aligned}
$$

Концентрации осколков удовлетворяют транспортным уравнениям

$$
\left[\frac{\partial}{\partial t}+u_{i} \frac{\partial}{\partial r_{i}}+\lambda^{\tau}\right] P^{\tau}(\mathbf{r}, t)=\int_{4 \pi} d \boldsymbol{\Omega} \sum_{g} \int_{0}^{m_{g}} v_{g}^{\tau}(m, \mathbf{r}, t) \Sigma_{g}^{f}(m, \mathbf{r}, t) \psi_{g}(m, \mathbf{\Omega}, \mathbf{r}, t) d m .
$$

Граничные условия выводятся из условий (3) аналогичным суммированием

$$
\left[\psi_{g}\left(m, \mathbf{\Omega}, \mathbf{r}_{\Gamma}\right)-\psi_{g}^{e n t}\left(m, \boldsymbol{\Omega}, \mathbf{r}_{\Gamma}\right)\right]_{\mathbf{n} \boldsymbol{\Omega}<0}=\int_{\mathbf{n} \mathbf{\Omega}^{\prime}>0} G_{g}\left(\boldsymbol{\Omega}^{\prime} \rightarrow \boldsymbol{\Omega}, m\right) \psi_{g}\left(m, \boldsymbol{\Omega}^{\prime}, \mathbf{r}_{\Gamma}\right) d \boldsymbol{\Omega}^{\prime} \text {. (39) }
$$


Осреднение уравнений ядерных реакций (7) выполняется с помощью (28), (29). Обсуждение уравнений (36)-(39) будет проведено позже, вместе с обсуждением уравнений метода лебеговых моментов.

Суммирование лебеговых сечений. Лебеговы макроскопические сечения есть суммы лебеговых микросечений с весом концентраций компонентов, аналогичные суммам (4), (5)

$$
\begin{aligned}
& \left\{\begin{array}{c}
1, \\
v_{h}^{y}\left(m^{\prime}, \mathbf{r}, t\right) W_{h \rightarrow g}^{y}\left(m^{\prime} \rightarrow m, \eta, \mathbf{r}, t\right)
\end{array}\right\} \times \Sigma_{h}^{y}\left(m^{\prime}, \mathbf{r}, t\right)= \\
& =\sum_{x, C} N_{C}(\mathbf{r}, t) \times\left\{\begin{array}{c}
1, \quad v_{C, h}^{x}\left(m^{\prime}\right) \\
v_{C, h}^{x}\left(m^{\prime}\right) w_{C, h \rightarrow g}^{x}\left(m^{\prime} \rightarrow m, \eta\right)
\end{array}\right\} \times \sigma_{C, h}^{x}\left(m^{\prime}\right), \\
& v_{g}^{\tau}(m, \mathbf{r}, t) \Sigma_{g}^{f}(m, \mathbf{r}, t) \chi_{g}^{\tau}(m, \mathbf{r}, t) \simeq \sum_{C} N_{C}(\mathbf{r}, t) v_{C, g}^{\tau}(m) \sigma_{C, g}^{f}(m) \chi_{C, g}^{\tau}(m) .
\end{aligned}
$$

Входящие в (40) микросечения вычисляются осреднением нейтронных микросечений $(8),(13)$ по лебеговым множествам $\omega(g, m)$

$$
\begin{aligned}
& \left\{1, \quad v_{C, g}^{x}(m)\right\} \times \sigma_{C, g}^{x}(m)=\frac{\partial}{\partial m} \int_{\omega(g, m)}\left\{1, v_{C}^{x}(E)\right\} \times \sigma_{C}^{x}(E) F(E) d E= \\
& =\sum_{k}\left[\left\{1, \quad v_{C}^{x}\left(E_{k}\right)\right\} \times \sigma_{C}^{x}\left(E_{k}\right) F\left(E_{k}\right) \frac{\partial E_{k}}{\partial m}\right]_{-}^{+}, \\
& v_{C, h}^{x}\left(m^{\prime}\right) \sigma_{C, h}^{x}\left(m^{\prime}\right) w_{C, h \rightarrow g}^{x}\left(m^{\prime} \rightarrow m, \eta\right)= \\
& =\frac{\partial^{2}}{\partial m \partial m^{\prime}} \int_{\omega(g, m)} d E \int_{\omega\left(h, m^{\prime}\right)} v_{C}^{x}\left(E^{\prime}\right) \sigma_{C}^{x}\left(E^{\prime}\right) w_{C}^{x}\left(E^{\prime} \rightarrow E, \eta\right) F\left(E^{\prime}\right) d E^{\prime}, \\
& v_{C, g}^{\tau}(m) \sigma_{C, g}^{f}(m) \chi_{C, g}^{\tau}(m)=\frac{\partial}{\partial m} \int_{\omega(g, m)} v_{C}^{\tau}(E) \sigma_{C}^{\tau}(E) \chi_{C}^{\tau}(E) F(E) d E .
\end{aligned}
$$

Напомним, что множества $\omega(g, m)$ строятся на основе среднего полного сечения $S^{t}(E)(18)$, вычисляемого для некоторого класса прикладных задач.

Аналитические формулы лебеговых сечений. Обсудим вопрос хранения лебеговых микросечений (41). Сечения зависят от переменной спектра $(g, m)$ и относительно слабо - от температуры вещества. Поэтому их можно хранить в файлах библиотеки сечений в виде аналитических формул, аналогично способу хранения исходных нейтронных сечений. 
Вследствие выполненного в разд.1 разбиения шкалы энергий на носители резонансов каждый носитель содержит резонансы некоторого компонента «C» и фоновое сечение остальных компонентов. Поэтому на носителе $\omega_{g}(C)$ лебеговы микросечения остальных компонентов $D \neq C$ можно разложить в быстро сходящиеся ряды Фурье по системе тригонометрических или степенных многочленов меры «m». Хранить требуется несколько первых коэффициентов разложений. Коэффициенты могут иметь слабую зависимость от температуры вещества.

Получим аналитические формулы для сечений компонента «C» на носителе своих резонансов $\omega_{g}(C)$. Предположим, что в пределах носителя резонансов и его окрестности резонансы размещены с постоянным шагом $d$, имеют одинаковую силу и полуширины $\Gamma, \Gamma^{x}$. В теории переноса фотонов модель одинаковых резонансов известна как приближение Эльзассера [44]

$$
E_{r}=E_{g}+\Delta+r d, \quad e=\left[E-E_{g}-\Delta\right] / d \quad \gamma_{g}=\Gamma / d .
$$

Здесь $E_{r}$ - положение резонанса с индексом «r», $E_{g}$ - центр носителя резонансов, $\Delta$ - сдвиг, $d$ - период, $e$ - безразмерная энергия $\gamma_{g}$ - безразмерная полуширина резонансов в окрестности носителя $\omega_{g}$.

Выполним в нейтронных сечениях (8) суммирование по резонансам, пользуясь следующими формулами (см., например, [45, с.527])

$$
\begin{aligned}
& \frac{1}{\pi} \sum_{r=-\infty}^{\infty} \frac{\gamma_{g}}{[e-r]^{2}+\gamma_{g}^{2}}=\frac{i}{2 \pi} \sum_{r=-\infty}^{\infty}\left[\frac{1}{e-r+i \gamma_{g}}-\frac{1}{e-r-i \gamma_{g}}\right]=\frac{\sinh 2 \pi \gamma_{g}}{\cosh 2 \pi \gamma_{g}-\cos 2 \pi e}, \\
& \frac{1}{\pi} \sum_{r=-\infty}^{\infty} \frac{e-r}{[e-r]^{2}+\gamma_{g}^{2}}=\frac{1}{2 \pi} \sum_{r=-\infty}^{\infty}\left[\frac{1}{e-r+i \gamma_{g}}+\frac{1}{e-r-i \gamma_{g}}\right]=\frac{\sin 2 \pi e}{\cosh 2 \pi \gamma_{g}-\cos 2 \pi e} .
\end{aligned}
$$

Таким образом, в пределах носителя резонансов $E \in \omega_{g}$

$$
\begin{aligned}
& \left\{\begin{array}{c}
\sigma_{C}^{t}(E) \\
\sigma_{C}^{x}(E)
\end{array}\right\}=\left\{\begin{array}{c}
\sigma_{g}^{p} \\
0
\end{array}\right\}+H_{g} \times\left\{\begin{array}{c}
p_{g} \theta_{g}(E)+q_{g} \chi_{g}(E) \\
u_{g}^{x} \theta_{g}(E), \quad x \neq t, e l
\end{array}\right\}, \quad \sigma_{C}^{e l}(E)=\sigma_{C}^{t}(E)-\sum_{x \neq e l} \sigma_{C}^{x}(E), \\
& \left\{\begin{array}{c}
\theta_{g}(E) \\
\chi_{g}(E)
\end{array}\right\}=\frac{1}{\cosh 2 \pi \gamma_{g}-\cos 2 \pi e} \times\left\{\begin{array}{c}
\sinh 2 \pi \gamma_{g} \\
\sin 2 \pi e
\end{array}\right\}, \quad \frac{1}{d} \int_{E}^{E+d}\left\{\begin{array}{c}
\theta_{g}\left(E^{\prime}\right) \\
\chi_{g}\left(E^{\prime}\right)
\end{array}\right\} d E^{\prime}=\left\{\begin{array}{l}
1 \\
0
\end{array}\right\},
\end{aligned}
$$$$
H_{g} \times\left\{1, p_{g}, q_{g}, u_{g}^{x}\right\}=4 \pi^{2} \lambda^{2} \sum_{l, J} n_{l J} g_{l J} \frac{\Gamma^{e l}}{d} \times\left\{1, \cos 2 \phi_{l}, \sin 2 \phi_{l}, \Gamma^{x} / \Gamma\right\} .
$$

Здесь $H_{g}$ - сила резонансов в единичном интервале энергий, $\theta_{g}(E)$ и $\chi_{g}(E)$ 
- симметричный и антисимметричный профили резонанса, определяемые на периоде сечения, $p_{g}$ и $q_{g}-$ коэффициенты фазового сдвига, $u_{g}^{x}-$ парциальный коэффициент реакции «х». Остальные величины пояснены в комментариях (8). На периоде $d$ уравнение $\sigma_{C}^{t}(E)=\sigma$ имеет два корня $E_{1}$ и $E_{2}$. Мера $\mu$ множества $\omega(g, \mu)=\left\{E: \sigma_{C}^{t}(E)<\sigma\right\}$ равна $\mu=d-\left[E_{2}-E_{1}\right]$. Положим $\mu / d \approx m / m_{g}$. В этой простой модели для лебеговых сечений можно найти аналитические формулы

$$
\begin{gathered}
\left\{\begin{array}{c}
\sigma_{C, g}^{t}(m) \\
\sigma_{C, g}^{x}(m)
\end{array}\right\} \approx\left\{\begin{array}{c}
\sigma_{g}^{p} \\
0
\end{array}\right\}+H_{g} \times\left\{\begin{array}{c}
p_{g} \theta_{g}(m)+q_{g} \chi_{g}(m) \\
u_{g}^{x} \theta_{g}(m), \quad x \neq t, e l
\end{array}\right\}, \quad 0 \leq m \leq m_{g}, \\
\theta_{g}(m)=\frac{\sinh 2 \pi \gamma_{g}}{\cosh 2 \pi \gamma_{g}+\cos \left(\pi m / m_{g}\right)}, \quad \chi_{g}(m) \approx-\frac{q_{g} \cos \left(\pi m / m_{g}\right)}{\left[1+p_{g}\right] \sinh 2 \pi \gamma_{g}}
\end{gathered}
$$

Такие же формулы получаются, если носитель резонансов содержит только левые или правые крылья резонансов (интервалы роста или падения сечения $\left.\sigma_{C}^{t}(E)\right)$. Формулы (42) распространяются на общий случай произвольных резонансов, если считать параметры $\sigma_{g}^{p}(m), p_{g}(m), q_{g}(m), u_{g}^{x}(m)$ функциями меры «m», а $\gamma_{g}(m, T), h_{g}(m, T)$ - функциями «m» и температуры. В виде (42) можно представлять и сечения отдельных нуклидов $A$, входящих в состав компонента $C$. Отметим, что формулы справедливы во всей области резонансов, включая участок неразрешенных резонансов.

Для хранения индикатрис $w_{C, h \rightarrow g}^{x}\left(m^{\prime} \rightarrow m, \eta\right)$ и кратностей продуктов реакций $v_{C, h}^{x}\left(m^{\prime}\right)$ можно пользоваться табличным представлением данных.

\section{3. Метод лебеговых моментов}

Численное решение уравнения (36) ищется на конечной сетке переменной нового спектра $\left(g, m_{i}\right), i=0, \ldots, N g, 1 \leq g \leq g g$. Поставим вопрос выбора оптимальной сетки $m_{i}$ при фиксированном общем числе узлов $\mathrm{Ng}$, которая передавала бы эволюцию поля нейтронов в объекте с минимальной (в определенном смысле) ошибкой. Проблема оптимизации сетки и проблема восстановления тока нейтронов в $E$-пространстве после нахождения лебегова тока нейтронов решается методом лебеговых моментов.

Разложение тока нейтронов. Будем искать решение уравнения переноса (36) на множествах $\omega(g, m) \subset \omega_{g}, 1 \leq g \leq g g$ в виде суммы главной функции $\psi_{N g}$ и малой поправочной функции $\varepsilon_{N g}$ 


$$
\begin{gathered}
\psi_{g}(m, \mathbf{\Omega}, \mathbf{r}, t)=\psi_{N g}(m, \mathbf{\Omega}, \mathbf{r}, t)+\varepsilon_{N g}(m, \mathbf{\Omega}, \mathbf{r}, t) \\
\psi_{N g}(m, \mathbf{\Omega}, \mathbf{r}, t)=\sum_{n=0}^{N g} \frac{P_{n}(\xi(m))}{d_{n}^{2}} \Psi_{g}^{(n)}(\mathbf{\Omega}, \mathbf{r}, t) \\
\Psi_{g}^{(n)}(\mathbf{\Omega}, \mathbf{r}, t)=\int_{-1}^{1} \rho(\xi) P_{n}(\xi) \psi_{g}(m, \mathbf{\Omega}, \mathbf{r}, t) d \xi, \quad \int_{-1}^{1} \rho(\xi) P_{n}(\xi) P_{k}(\xi) d \xi=d_{n}^{2} \delta_{n k} . \\
\xi(m)=\frac{e^{-S_{0 g} L_{g}}+e^{-S_{g} L_{g}}-2 e^{-S(m) L_{g}}}{e^{-S_{0 g} L_{g}}-e^{-S_{g} L_{g}}, \quad 0 \leq m \leq m_{g}, \quad S_{0 g} \leq S(m) \leq S_{g},} \quad-1 \leq \xi(m) \leq 1 .
\end{gathered}
$$

Главная функция есть отрезок разложения лебегова тока нейтронов $\psi_{g}(m)$ в ряд по некоторой системе многочленов $P_{n}(\xi)(n=0,1, \ldots)$, ортогональных на интервале $-1 \leq \xi(m) \leq 1 ; N g$ - порядок разложения, $\rho(\xi)$ - весовая функция системы многочленов, $d_{n}$ - нормировочные множители. Коэффициенты разложения $\Psi_{g}^{(n)}(\boldsymbol{\Omega}, \mathbf{r}, t)$ будем называть лебеговыми моментами. Аргумент многочленов $\xi(m)=\xi(S(m))$ есть сложная функция, которая монотонно возрастает по аргументу $S=S(m)$, не убывает в зависимости от $m$ и принимает значения $\xi(0)=-1, \xi\left(m_{g}\right)=1$ на границах интервала. В исходном $E$-пространстве разложению (43) соответствует следующее разложение тока нейтронов на носителе резонансов

$$
\begin{array}{ll}
\varphi(E, \boldsymbol{\Omega}, \mathbf{r}, t)=F(E) \sum_{n=0}^{N g} \frac{P_{n}(\xi(E))}{d_{n}^{2}} \Psi_{g}^{(n)}(\mathbf{\Omega}, \mathbf{r}, t)+\varepsilon_{N g}(E, \mathbf{\Omega}, \mathbf{r}, t), & E \in \omega_{g}, \\
\xi(E)=\xi(S(E))=\frac{e^{-S_{0 g} L_{g}}+e^{-S_{g} L_{g}}-2 e^{-S(E) L_{g}}}{e^{-S_{0 g} L_{g}}-e^{-S_{g} L_{g}}}, & 1 \leq g \leq g g,
\end{array}
$$

в чем несложно убедиться, подставив (45) в (25). (Сp. (45) с (20), (21).)

Если подставить (44) в (43) и привести подобные члены, то главную часть тока нейтронов $\psi_{N g}$ (или $\varphi_{N g}$ ) можно записать в виде линейной комбинации экспонент: $\exp \left(-n S(u) L_{g}\right), 0 \leq n \leq N g, u=E, m$. Показатель $n$ задает парциальную скорость выбивания нейтронов из пучка данной экспонентой; $S(u)(u=E, m)$ - среднее сечение $(18) ; L=L_{g}$ - подбираемый для задачи (класса задач) размерный параметр, имеющий порядок размера объекта. Группировка экспонент в многочлены - это способ ортогонализации базисных функций. Включение в базис новой экспоненты эквивалентно добавлению в $\psi_{N g}$ еще одной гармоники высшего порядка. Выбор экспонент 
в качестве базисных функций продиктован формой решения уравнения переноса частиц (21), которая суть сумма некоторых экспонент.

Также подходящими базисными функциями для описания обращения резонансов являются рациональные функции $1 /\left[1+S(u) L_{g}\right]^{n}, n=0,1, \ldots, u=E, m$. Для перехода к этой системе достаточно выполнить аппроксимацию экспоненты $\exp \left(-S(u) L_{g}\right) \approx 1 /\left[1+S(u) L_{g}\right]$. Тогда аргумент многочленов (44) в разложениях (43), (45) заменяется аргументом

$$
\xi(u)=\xi(S(u))=1-2 \frac{S_{g}-S(u)}{S_{g}-S_{0 g}} \frac{1+S_{0 g} L_{g}}{1+S(u) L_{g}}, \quad \begin{gathered}
u=E, m . \\
u=1,
\end{gathered}
$$

Возможно, существуют и другие удобные системы базисных функций для описания эффектов обращения резонансов. Название лебеговы моменты отражает зависимость базисных функций разложений (43), (44) и (43), (46) от величины сечения взаимодействия нейтронов с веществом $S(u)(u=E, m)$.

Пусть главная часть разложений (43), (45) сходится к току нейтронов в точке $(\boldsymbol{\Omega}, \mathbf{r}, t)$ на носителях резонансов $E \in \omega_{g}$ при увеличении $\mathrm{Ng}$ и уменьшении ширины носителей $K_{g}$ (см. (15)), например, в норме $L_{2}:\left\|\varepsilon_{N g}(u)\right\|_{2} \rightarrow 0$, $u=E, m \in \omega_{g}$. Скорость сходимости зависит от того, правы ли мы в выборе базисных функций (44), (46) для описания обращения резонансов, от выбора масштабного параметра $L_{g}$ в этих функциях, от решаемой задачи: материалов и геометрии объекта.

Тогда малая ошибка, допущенная при выводе уравнения переноса (36) для лебегова тока нейтронов, по мере сходимости быстро стремится к нулю. В самом деле, главная функция разложения $\varphi_{N g}(E)$ зависит от энергии $E$ только через среднее макросечение $S(E)$. Поэтому дисперсия $D\left(m, \varepsilon_{N g}\right)$ во втором неравенстве (31) стремится к нулю. Это ускоряет итоговую сходимость уравнения (36) и уравнения (1) в норме $L_{2}$ вследствие одновременного выполнения неравенств (31). Скорость сходимости много выше скорости сходимости уравнений многогруппового приближения к (1).

Восстановление спектра нейтронов. Если известны лебеговы моменты $\Psi_{g}^{(n)}(\boldsymbol{\Omega}, \mathbf{r}, t) \quad(0 \leq n \leq N g, 1 \leq g \leq g g)$, то ток нейтронов внутри объекта в приближении $\varepsilon_{N g} \approx 0$ восстанавливается по формулам (43), (45) как в $(g, m)$ пространстве, так и в $E$-пространстве (если требуется).

Вычисление интегралов. Введение лебеговых моментов приводит к появлению дополнительных лебеговых сечений, упрощающих расчеты функционалов поля нейтронов - тока нейтронов на носителе резонансов $g$; скоростей ухода нейтронов с носителя; скоростей появления мгновенных нейтронов, предшественников (см. (2)) и нуклидов (см. (7)) в реакциях «x» 


$$
\begin{aligned}
& \left\{\begin{array}{c}
\varphi_{g}(\mathbf{\Omega}, \mathbf{r}, t), \quad I_{g}^{x}(\mathbf{\Omega}, \mathbf{r}, t) \\
J_{g}^{x}(\mathbf{\Omega}, \mathbf{r}, t)
\end{array}\right\}=\int_{\omega_{g}}\left\{\begin{array}{cc}
1, & \Sigma^{x}(E, \mathbf{r}, t) \\
v^{x}(E, \mathbf{r}, t) \Sigma^{x}(E, \mathbf{r}, t)
\end{array}\right\} \times \varphi(E, \mathbf{\Omega}, \mathbf{r}, t) d E \approx \\
& \approx \int_{0}^{m_{g}}\left\{\begin{array}{c}
1, \quad \Sigma_{g}^{x}(m) \\
v_{g}^{x} \Sigma_{g}^{x}(m)
\end{array}\right\} \times \psi_{N g}(m, \mathbf{\Omega}) d m=\sum_{n=0}^{N g} \frac{F_{g}^{(n)}}{d_{n}^{2}}\left\{\begin{array}{c}
1, \quad \Sigma_{g}^{x(n)}(\mathbf{r}, t) \\
v_{g}^{x(n)} \Sigma_{g}^{x(n)}(\mathbf{r}, t)
\end{array}\right\} \times \Psi_{g}^{(n)}(\mathbf{\Omega}, \mathbf{r}, t),
\end{aligned}
$$

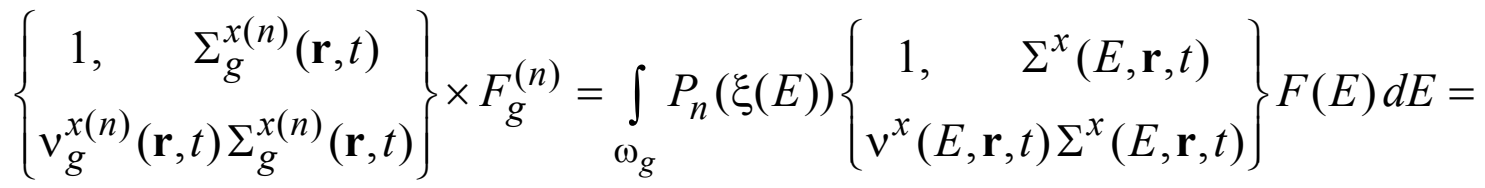

$$
\begin{aligned}
& =\int_{0}^{m_{g}} P_{n}(\xi(m)) \times\left\{\begin{array}{cc}
1, & \Sigma_{g}^{x}(m, \mathbf{r}, t) \\
v_{g}^{x}(m, \mathbf{r}, t) \Sigma_{g}^{x}(m, \mathbf{r}, t)
\end{array}\right\} d m .
\end{aligned}
$$

Здесь $F_{g}^{(n)}=F_{g}^{(n)}\left(L_{g}\right)$ - вес лебегова момента порядка $n$ на носителе $g$, $\Sigma_{g}^{x(n)}(\mathbf{r}, t)$ и $v_{g}^{x(n)}(\mathbf{r}, t)$ - макросечение и выход частиц порядка $n$ в реакциях «x». Вес момента нулевого порядка равен интегралу от весового спектра $F_{g}^{(0)}=F_{g}$. Макросечение нулевого порядка $n=0$ соответствует макросечению многогруппового приближения. Дифференциальные скорости прихода мгновенных нейтронов $Q_{g}^{x}(m, \boldsymbol{\Omega}, \mathbf{r}, t), q_{N g}^{x}(\psi)(32)$ в множество $E \in \omega(g, m)$ и в единичный интервал меры «m» записываются в виде:

$$
\begin{aligned}
& Q_{g}^{x}(m, \mathbf{\Omega}, \mathbf{r}, t) \approx \int_{0}^{m} q_{N g}^{x}(\psi) d m, \quad 0 \leq m \leq m_{g}, \\
& q_{N g}^{x}(\psi)=\int_{4 \pi} \frac{d \mathbf{\Omega}^{\prime}}{2 \pi} \sum_{h} \sum_{n=0}^{N} \frac{F_{h}^{(n)}}{d_{n}^{2}} \Sigma_{h}^{x(n)}(\mathbf{r}, t) v_{h}^{x(n)}(\mathbf{r}, t) W_{h \rightarrow g}^{x(n)}(m, \eta, \mathbf{r}, t) \Psi_{h}^{(n)}\left(\mathbf{\Omega}^{\prime}, \mathbf{r}, t\right) \\
& v_{h}^{x(n)}(\mathbf{r}, t) W_{h \rightarrow g}^{x(n)}(m, \eta, \mathbf{r}, t) \Sigma_{h}^{x(n)}(\mathbf{r}, t) F_{h}^{(n)}= \\
& =\frac{\partial}{\partial m} \int_{\omega(g, m)} d E \int_{\omega_{h}} P_{n}\left(\xi^{\prime}\left(E^{\prime}\right)\right) \Sigma^{x}\left(E^{\prime}, \mathbf{r}, t\right) v^{x}\left(E^{\prime}, \mathbf{r}, t\right) W^{x}\left(E^{\prime} \rightarrow E, \eta, \mathbf{r}, t\right) F\left(E^{\prime}\right) d E^{\prime}, \\
& \sum_{g} \int_{0}^{m_{g}} d m \int_{-1}^{1} W_{h \rightarrow g}^{x(0)}(m, \eta, \mathbf{r}, t) d \eta=1 \quad(n=0)
\end{aligned}
$$

где $W_{h \rightarrow g}^{x(n)}(m, \eta, \mathbf{r}, t)\left(\eta=\mathbf{\Omega} \mathbf{\Omega}^{\prime}\right)$ - лебегов момент порядка $n$ индикатрисы реакций «х» (не путать с угловым моментов индикатрисы, см. (9)). 
Моменты сечений (48), (50) вычисляются через лебеговы микросечения компонентов по формулам (40), (41).

Расчет лебеговых моментов. Моменты распределения можно вычислять, следуя их формальному определению (43) с помощью неоптимизированных квадратурных формул: прямоугольников, трапеций и т.д. Кроме примитивного способа, применяются два прямых метода расчета моментов. Первый метод состоит в формулировке системы уравнений для моментов и еe решении. Второй метод использует оптимизированные квадратурные формулы типа формул Гаусса. Решение уравнения переноса частиц ищется на конечной сетке, состоящей из $N+1$-го узла квадратурной формулы. Эта сетка является оптимальной в том смысле, что предоставляет возможность точно вычислить $M(M \leq N)$ степенных моментов, если распределение непрерывно по данной переменной, $M$ - параметр качества формулы. В случае $M=N$ оба метода эквивалентны и сетка из $N+1$-го узла суть необходимое, но не достаточное условие для точного расчета моментов распределения частиц в объекте. Для расчета лебеговых моментов далее рассматривается вариант квадратурных формул.

Замечание. В задачах переноса частиц для расчета угловых моментов распределений частиц по направлениям полета $\Omega$ применяются прямые методы, которые известны под названиями соответственно - метод сферических гармоник и метод дискретных ординат Вика-Чандрасекара, а также оптимизированный $\mathrm{Sn}$-метод Карлсона (в многомерных задачах).

Квадратурной формулой Гаусса-Кристоффеля называется формула приближенного вычисления интегралов [46]

$$
\int_{-1}^{1} \rho(\xi) Y(\xi) d \xi \approx \sum_{i=0}^{N} \lambda_{i} Y\left(\xi_{i}\right), \quad \sum_{i=0}^{N} \lambda_{i}=\int_{-1}^{1} \rho(\xi) d \xi .
$$

Здесь $Y(\xi)$ - интегрируемая функция из некоторого класса функций, $\rho(\xi)-$ весовая функция, выделяющая особенности поведения функций этого класса на интервале $-1 \leq \xi \leq 1 ; Y\left(\xi_{i}\right)$ - значения интегрируемой функции в узлах формулы $\xi_{i}(i=0,1, \ldots, N)$. Узлы есть корни уравнения $P_{N+1}\left(\xi_{i}\right)=0$, где $P_{N+1}$ принадлежит системе ортогональных многочленов на интервале $-1 \leq \xi \leq 1$ с весом $\rho(\xi)$ (см. (43)). Веса формулы $\lambda_{i}$, называемые коэффициентами Кристоффеля, вычисляются как

$$
\frac{1}{\lambda_{i}}=\frac{a_{N} P_{N}\left(\xi_{i}\right)}{a_{N+1} d_{N}^{2}} \frac{d P_{N+1}\left(\xi_{i}\right)}{d \xi}=\sum_{n=0}^{N} \frac{P_{n}^{2}\left(\xi_{i}\right)}{d_{n}^{2}}, \quad i=0,1, \ldots, N
$$


где $a_{N}-$ коэффициент при старшей степени многочлена $P_{N}$. Узлы и веса подобраны так, что приближенное равенство (51) становится точным равенством для любого многочлена $Y(\xi)$ степени не выше $2 N+1$.

Рассмотрим разложение (43). Лебегов ток $\psi_{g}(m)$ и его главная часть $\psi_{N g}(m)$ имеют одинаковые младшие моменты $\Psi_{g}^{(n)}, 0 \leq n \leq N$. Так как степень многочлена $Y(\xi)=P_{n}(\xi) \psi_{N g}(m)$ при $0 \leq n \leq N$ не превышает $2 N$, то его подстановка в (51) дает точную формулу вычисления моментов:

$$
\Psi_{g}^{(n)}(\boldsymbol{\Omega}, \mathbf{r}, t)=\int_{-1}^{1} \rho(\xi) P_{n}(\xi) \psi_{g}(m(\xi), \mathbf{\Omega}, \mathbf{r}, t) d \xi=\sum_{i=0}^{N} \lambda_{i} P_{n}\left(\xi_{i}\right) \psi_{g}\left(m_{i}, \mathbf{\Omega}, \mathbf{r}, t\right) .
$$

Если значения моментов известны (например, с предыдущей итерации), то скорость прихода нейтронов $q_{g}^{s}(\psi)$, входящую в уравнение (36), можно вычислять по формуле (49) вместо формулы (33).

Для того чтобы пользоваться (52), решение $\psi_{g}\left(m_{i}, \mathbf{\Omega}, \mathbf{r}, t\right)$ уравнения переноса нейтронов (36) ищется на оптимальной сетке $m_{i}=m\left(\xi_{i}\right)(i=0,1, \ldots, N)$, или на сетке, ее включающей, или на близкой сетке, допускающей интерполяцию высокой точности.

Для нахождения узлов оптимальной сетки $m_{i}$ обратим (44) и (46)

$$
\begin{array}{ll}
S_{i}\left(m_{i}\right) L_{g}=\ln \frac{2}{\left[1-\xi_{i}\right] e^{-S_{0 g} L_{g}}+\left[1+\xi_{i}\right] e^{-S_{g} L_{g}}}, & \text { экспонент, } \\
S_{i}\left(m_{i}\right)=\frac{\left[1+\xi_{i}\right] S_{g}+\left[1-\xi_{i}\right] S_{0 g}+2 S_{0 g} S_{g} L_{g}}{2+\left[1-\xi_{i}\right] S_{g} L_{g}+\left[1+\xi_{i}\right] S_{0 g} L_{g}}, & \text { базис рациональных } \\
\text { функций. }
\end{array}
$$

Пример. Пусть для ортогонализации базисных функций спектра нейтронов (44), (46) в разложении (43) используются многочлены Чебышева, ортогональные на интервале $-1 \leq \xi \leq 1$ с весом $\rho(\xi)=\left[1-\xi^{2}\right]^{-1 / 2}$ :

$$
P_{n}(\xi)=\cos (n \arccos \xi), \quad \int_{-1}^{1} \frac{P_{n}(\xi) P_{m}(\xi)}{\sqrt{1-\xi^{2}}} d \xi=d_{n}^{2} \delta_{n m}, \quad d_{n}^{2}=\frac{\pi}{2}\left[1+\delta_{n 0}\right] .
$$

Узлы, значения многочленов в узлах и веса квадратурной формулы (51):

$$
\xi_{i}=\cos \frac{\pi[2 i+1]}{2[N+1]}, \quad P_{n}\left(\xi_{i}\right)=\cos \frac{\pi n[2 i+1]}{2[N+1]}, \quad \lambda_{i}=\frac{\pi}{N+1}, \quad 0 \leq i, n \leq N .
$$

Формула вычисления лебеговых моментов (52) принимает вид: 


$$
\Psi_{g}^{(n)}(\boldsymbol{\Omega}, \mathbf{r}, t)=\frac{\pi}{N+1} \sum_{i=0}^{N} \cos \frac{\pi n[2 i+1]}{2[N+1]} \psi_{g}\left(m_{i}, \mathbf{\Omega}, \mathbf{r}, t\right) .
$$

Уравнения (53) для нахождения оптимальной сетки $m_{i}$ :

$$
\begin{aligned}
& S_{i}\left(m_{i}\right) L_{g}=-\ln \left(e^{-S_{0 g} L_{g}} \sin ^{2} \frac{\pi[2 i+1]}{4[N+1]}+e^{-S_{g} L_{g}} \cos ^{2} \frac{\pi[2 i+1]}{4[N+1]}\right), \quad \begin{array}{l}
\text { базис } \\
\text { экспонент; }
\end{array} \\
& S_{i}\left(m_{i}\right)=\frac{S_{g} \cos ^{2} \frac{\pi[2 i+1]}{4[N+1]}+S_{0 g} \sin ^{2} \frac{\pi[2 i+1]}{4[N+1]}+S_{0 g} S_{g} L_{g}}{1+S_{g} L_{g} \sin ^{2} \frac{\pi[2 i+1]}{4[N+1]}+S_{0 g} L_{g} \cos ^{2} \frac{\pi[2 i+1]}{4[N+1]}}, \quad \begin{array}{l}
\text { базис рациональных } \\
\text { функций. }
\end{array}
\end{aligned}
$$

Разрешение этих уравнений (или общих уравнений (53)) относительно $m_{i}$ $(i=0,1, \ldots, N)$ выполняется на этапе подготовки данных для транспортного расчета вместе с подготовкой лебеговых макросечений. Макросечения можно вычислять непосредственно по файлам библиотек ядерных данных либо по файлам библиотеки лебеговых нейтронных микросечений.

\section{Обсуждение}

Сравнение с многогрупповым приближением. В области резонансов нейтронных сечений уравнения многогруппового приближения имеют лишь модельное соответствие с исходными уравнениями переноса нейтронов (1)(3), (7) на качественном уровне. В самом деле, рассмотрим «точные» групповые константы, вычисляемые по схеме Римана с весом тока нейтронов. Точные групповые константы зависят от направления полета нейтронов $\Omega$. Их вариации по $\Omega$ могут достигать десятков и сотен процентов, особенно вблизи границ раздела материалов, где ток нейтронов (21) сильно меняется по обоим аргументам $E, \Omega$ (см., например, [47]). Групповые константы многогруппового приближения $[34,35,38]$ не зависят от $\boldsymbol{\Omega}$. Они теряют эту зависимость, т.к. при осреднении истинный ток нейтронов заменяется приближенным изотропным весовым спектром.

Уравнения метода лебеговского осреднения и метода лебеговых моментов (36)-(39), (49), (52) аппроксимируют исходные уравнения переноса нейтронов (1)-(3), (7) с высокой точностью. Лебеговы сечения теряют мало информации о нейтронных сечениях и имеют точность, сопоставимую с точностью данных в библиотеках ядерных данных. Как и исходные нейтронные сечения, они не зависят от направления полета нейтронов $\Omega$.

Осредненные уравнения описывают такие эффекты в спектрах ней- 
тронов, как интерференция потенциального и резонансного рассеяния; увеличение выхода нейтронов деления $v^{f}(E)$ в ядрах резонансов; обращение узких и широких резонансов; блок-эффект - уменьшение резонансной самоэкранировки нейтронного поля вблизи границ раздела материалов; пространственная неоднородность выгорания топлива и образования нуклидов в ядерном реакторе. Возможность приближенно восстанавливать спектр нейтронов делает метод лебеговых моментов хорошим инструментом для численной поддержки нейтронной диагностики объектов.

Численное решение. Сечения уравнений и ток нейтронов имеют высокую монотонность по переменной спектра $(g, m)$. Уравнения имеют структуру, аналогичную структуре уравнений в $E$-пространстве. Поэтому их численное решение можно выполнять на редкой сетке, применяя известные методы расчета пространственно-углового распределения нейтронов: прямые методы решения уравнения переноса (Sn-метод, метод характеристик), метод сферических гармоник и метод Монте-Карло.

Метод моментов нулевого порядка есть многогрупповое приближение с группами - носителями резонансов. Это обеспечивает плавный переход от метода моментов $N>0$ к многогрупповому приближению $N=0$ при выходе из области резонансов в область медленной вариации сечений.

Переходы нейтронов вниз и вверх по переменной $m$ в пределах носителя резонансов $(h=g)$ и переходы вниз и вверх по индексу носителей резонансов требуют выполнения итераций. Эти итерации аналогичны итерациям, которые выполняются в области термализации нейтронов при использовании многогруппового приближения. В методе лебеговых моментов итерации сводятся к нахождению лебеговых моментов.

Применение метода требует написания компьютерного кода для подготовки лебеговых нейтронных сечений. Сечения вычисляются осреднением микроскопических сечений по несколько более сложному алгоритму, чем алгоритм осреднения в группы.

Проверка эффективности метода. На момент написания статьи проверка эффективности метода лебеговых моментов в численном моделировании задач переноса нейтронов не проводилась. Результаты тестовых расчетов на примере задач переноса теплового излучения в горячем газе приведены в [12].

Эффект обращения резонансов характерен не только для спектров нейтронов, но и для широких спектральных распределений фотонов, в том числе для «собственных» фотонов теплового излучения, испускаемых неоднородно нагретым газом или плазмой. Вследствие сильной зависимости мощ- 
ности тепловых источников от температуры $\sim T^{4}$ спектр излучения в крыльях линий формируется наиболее горячими источниками объекта. Напротив, в ядрах линий спектр излучения формируется относительно холодными локальными тепловыми источниками. Даже небольшие вариации температуры, например, в три раза, приводят к сильному обращению линий.

Решение тестовых задач, в том числе для гетерогенных объектов, показало следующее. Разложение (43) быстро сходится к точному решению. В случае группировки базисных функций в многочлены Чебышева достаточно ограничиться учетом $N=4 \div 6$ гармоник. Разложение с группировкой в многочлены Лежандра сходится несколько медленнее $-N=6 \div 8$. Для анализа скорости сходимости кроме нормы ошибки оценивалось распределение «энергии» по гармоникам разложения

$$
\int_{-1}^{1} \psi_{g}^{2}(m(\xi), \mathbf{\Omega}, \mathbf{r}, t) d \xi \approx \int_{-1}^{1} \psi_{N g}^{2}(m(\xi), \mathbf{\Omega}, \mathbf{r}, t) d \xi=\sum_{n=0}^{N} \frac{1}{d_{n}^{2}}\left[\Psi_{g}^{(n)}(\mathbf{\Omega}, \mathbf{r}, t)\right]^{2} .
$$

Весьма хорошим свойством метода оказалось то, что скорость сходимости слабо зависит от изменений масштабного параметра базисных функций $L_{g}$ - характерного размера объекта, в довольно широком диапазоне. Параметр уменьшался и увеличивался относительно некоторого оптимального значения до 10 раз. Ошибка и «энергия» хвоста разложения начинали расти только в непосредственной близости от границ указанного диапазона.

Оценка числа расчетных точек. Пусть для разделения резонансной структуры нейтронных сечений область резонансов разбивается на $30 \div 200$ носителей резонансов - в зависимости от типа решаемых задач и требований к точности. Использование в каждом носителе от 4 до 6 лебеговых моментов дает от 100 до 1000 расчетных точек $(g, m)$. В результате решения осредненных уравнений может быть достигнута точность описания спектра нейтронов, сопоставимая с точностью детальных расчетов методом МонтеКарло и точностью ядерных данных.

\section{СПИСОК ЛИТЕРАТУРЫ}

1. Николаев М.Н., Филиппов В.В. Измерение параметров резонансной структуры сечений некоторых элементов в области энергий нейтронов 0.3-2.7 МэВ // Атомная энергия, 1963, т. 15(6), с. 493-498.

Nikolaev M.N., Filippov V.V. Measurement of the resonance structure parameters of the total cross sections of some elements in the neutron energy range 0.3-2.7 MeV // Soviet Atomic Energy, 1963, v.15(6), p. 1281-1286. 
2. Николаев М.Н., Игнатов А.А., Исаев Н.В., Хохлов В.Ф. Метод подгрупп для учета резонансной структуры сечений в нейтронных расчетах. Ч. 1 // Атомная энергия, 1970, т. 29(1), c. 11-16.

Nikolaev M.N., Ignatov A.A., Isaev N.V., Kokhlov V.F. The method of subgroups for considering the resonance structure of the cross sections in neutron calculations. P.1 // Soviet Atomic Energy, 1970, v. 29(1), p. 689-695.

3. Николаев М.Н., Игнатов А.А., Исаев Н.В., Хохлов В.Ф. Метод подгрупп для учета резонансной структуры сечений в нейтронных расчетах. Ч. 2 // Атомная энергия, 1971, т. 30(5), c.426-430.

Nikolaev M.N., Ignatov A.A., Isaev N.V., Kokhlov V.F. The method of subgroups for considering the resonance structure of cross sections in neutron calculations // Soviet Atomic Energy, 1971, v. 30(5), p. 528-533.

4. Levitt L.B. The probability table method for treating unresolved neutron resonances in Monte Carlo calculations // Nuclear Sci. Eng., 1972, v. 49(4), p. 450-457.

5. Cullen D.E. Application of the probability table method to multigroup calculations of neutron transport // Nuclear Sci. Eng., 1974, v. 55, p. 387-400.

6. Cullen D.E. Nuclear data preparation / Handbook of nuclear engineering. V. I. Nuclear engineering fundamentals. Ed. Cacuci D.G. - NY: Springer, 2010, p. 282-425.

7. Цветкова И.Л., Шильков А.В. Осреднение уравнения переноса в резонансно поглощающей среде // Матем. моделирование, 1989, т.1(1), с.91-100.

Tsvetkova I.L., Shilkov A.V. Osrednenie uravneniia perenosa v rezonansno pogloshchaiushchei srede // Matem. modelirovanie, 1989, t.1(1), s.91-100.

8. Шильков А.B. Методы осреднения сечений и энергетического спектра в задачах переноса нейтронов // Матем. моделирование, 1991, т.3(2), с.63-81.

Shilkov A.V. Metody osredneniia sechenii i energeticheskogo spektra v zadachakh perenosa neitronov // Matem. modelirovanie, 1991, t.3(2), s.63-81.

9. Shilkov A.V. Generalized multigroup approximation and Lebesgue averaging method in particle transport problems // Transp. Theory and Stat. Physics, 1994, v.23(6), p.781-814.

10. Шильков А.В., Гериев М.Н. Верификация метода лебеговского осреднения // Матем. моделирование, 2015, т. 27(8), с. 13-31.

Shilkov A.V., Gertsev M.N. Verification of the Lebesgue averaging method // Math. Models and Comp. Simulations, 2015, v. 27(8), p. 13-31.

11. Аристова Е.Н., Гериев М.Н., Шильков А.В. Метод лебеговского осреднения в серийных расчетах атмосферной радиации // ЖВМ и МФ, 2017, т.57(6), с. 1033-1047.

Aristova E.N., Gertsev M.N., Shilkov A.V. Lebesgue averaging method in serial computations of atmospheric radiation // Comp. Math. \& Math. Phys., 2017, v.57(6), p.1022-1035.

12. Шильков A.B. Моментный метод лебеговой агрегации и восстановления спектра в проблемах переноса частиц // Матем. моделирование, 2016, т. 28(11), с. 33-54.

Shilkov A.V. The moment method of Lebesgue aggregation and spectrum recovery in particle transport problems // Math. Models and Comp. Simul., 2017, v. 9(3), p. 263-280.

13. Кондратьев К.Я. О поглощении длинноволновой радиации в атмосфере // Метеорология и гидрология, 1947, №6, с. 3-12.

Kondratev K.Ia. O pogloshchenii dlinnovolnovoi radiatsii v atmosfere // Meteorologiia i gidrologiia, 1947, №6, s. 3-12.

14. Кондратьев К.Я. Перенос длинноволнового излучения в атмосфере. - М.: ГТИ, 1950.

Kondratev K.Ia. Perenos dlinnovolnovogo izlucheniia v atmosfere. - M.: GTI, 1950.

15. Cullen D.E., Pomraning G.C. The multiband method in radiative transfer calculations // 
Quant. Spectrosc. and Rad. Transfer, 1980, v.24, p. 97-117.

16. Arking A.A., Grossman K. The Influence of line shape and band structure on temperatures in planetary atmospheres // J. Atmosph. Sci., 1972, v. 29, p. 937-949.

17. Domoto D.A. Frequency integration for radiative transfer problems involving homogeneous non-gray gases: the inverse transmission function // Quant. Spectrosc. \& Rad. Transfer., 1974, v.14, p. 935-942.

18. Lacis A.A., Oinas $V$. A description of the correlated k-distribution method for modeling nongray gaseous absorption. Thermal emission and multiple scattering in vertically inhomogeneous atmospheres // J. Geoph. Research, 1991, v. 96(D5), p. 9027-9063.

19. Fu Q., Liou K.N. On the correlated k-distribution method for radiative transfer in nonhomogeneous atmospheres // J. Atmosph. Sci., 1992, v.49, p.2139-2156.

20. Mlawer E.J., Taubman S.J., Brown P.D., Iacono M.J., Clough S.A. Radiative transfer for inhomogeneous atmospheres: RRTM, a validated correlated-k model for the longwave // J. Geoph. Research, 1997, v.102(D14), p.16,663-16,682.

21. Modest M.F. Narrow-band and full-spectrum k-distributions for radiative heat transfer correlated-k vs. scaling approximation // Quant. Spectrosc. \& Rad. Transfer, 2003, v.76(1), p.69-83.

22. Ambartzumian $V$. The effect of the absorption lines on the radiative equilibrium of the outer layers of the stars // Уч. зап. Ленингр. универс., сер. Мат. наук (Астрономия), 1936, т. 6(1), c.7-18. [in English].

Uchenye zapiski Leningr. universiteta, ser. Mat. nauk (Astronom.), 1936, t. 6(1), s.7-18.

23. Stewart J.C. Non-grey radiative transfer// Quant. Spectr. \& Rad. Transfer, 1964, v.4, p. 723-729.

24. Гольдин В.Я., Четверушкин Б.Н. Методы решения одномерных задач радиационной газовой динамики // ЖВМ и МФ, 1972, т. 12(4), с. 990-1001.

Gol'din V.Ya., Chetverushkin B.N. Methods of solving one-dimentional problems of radiation gas dynamics // USSR Comp. Math. \& Math. Physics, 1972, v.12(4), p.177-189.

25. Кривцов В.М. Об одном подходе к расчету селективного излучения // ЖВМ и МФ, 1974, т. 14(6), с.1595-1599.

Krivtsov V.M. Ob odnom podkhode k raschetu selektivnogo izlucheniia // Zh. Vych. mat. i mat. fiziki, 1974, t. 14(6), s.1595-1599.

26. Block R.C., Danon Y., Gunsing F., Haight R.C. Neutron cross section measurements / Handbook of nuclear engineering. V.I. Nuclear engineering fundamentals. Ed. Cacuci D.G. - NY.: Springer, 2010, p. 3-81.

27. Obložinsky P., Herman M., Mughabghab S.F. Evaluated nuclear data / Ibid, p. 86-187.

28. Frohner F. Evaluation and analysis of nuclear resonance data. - JEFF Rep.18, OECD/ NEA, 2000, $124 \mathrm{p}$.

29. Nuclear Energy Agency. Evaluated nuclear data library descriptions.

30. ENDF/B-VII.1 Evaluated Nuclear Data Library. ENDF-6 Formats manual. Data formats \& procedures for the Evaluated Nucl. Data Files ENDF/B-VI, ENDF/B-VII \& ENDF/B-VIII. Eds. A. Trkov, M. Herman and D.A. Brown. - Brookhaven Nation. Lab. Upton, NY, 2018, Rep. BNL-203218-2018-INRE, Rev.215, 418 p.

31. РОСФОНД - РОСсийская библиотека Файлов Оцененных Нейтронных Данных. ROSFOND - Russian Library of Evaluated Neutron Data.

32. Nuclear Energy Agency. JEFF-3.2 evaluated data library - Neutron data.

33. JENDL - Japanese Evaluated Nuclear Data Library.

34. MacFarlane R.E., Kahler A.C. Methods for processing ENDF/B-VII with NJOY // Nuclear 
Data Sheets, 2010, v. 111(12), p. 2739-2890.

35. MacFarlane R.E., Muir D.W., Boicourt R.M., Kahler A.C., Conlin J.L., Lloyd J. The NJOY nuclear data processing system, Vers. 2016 - USA, LANL report LA-UR-17-20093, 2016.

36. MacFarlane R.E. Neutron slowing down and thermalization / Handbook of nuclear engineering. V.I. Nuclear engin. fundam. Ed. Cacuci D.G. - NY: Springer, 2010, p.181-277.

37. Вейнберг А., Вигнер Е. Физическая теория ядерных реакторов. - М.: ИЛ, 1961.

Weinberg A.M., Wigner E.P. The physical theory of neutron chain reactors. - The University of Chicago Press, 1958.

38. Кощеев В.Н., Мантуров Г.Н., Николаев М.Н., Цибуля А.М. Библиотека групповых констант БНАБ-РФ для расчетов реакторов и защиты // Изв. вузов. Ядерная энергетика, 2014, N 3, с. 93-101.

Koshcheev V.N., Manturov G.N., Nikolaev M.N., Tsibulia A.M. Biblioteka gruppovykh konstant BNAB-RF dlia raschetov reaktorov i zashchity // Izv. vuzov. Iadernaia energetika, 2014, N 3, s. 93-101.

39. Абагян Л.П., Базазяни Н.О., Бондаренко И.И., Николаев М.Н. Групповые константы для расчетов ядерных реакторов. - М.: Атомиздат, 1964.

Abagian L.P., Bazaziants N.O., Bondarenko I.I., Nikolaev M.N. Gruppovye konstanty dlia raschetov iadernykh reaktorov. - M.: Atomizdat, 1964.

40. Amaldi E., Fermi E. On the absorption and the diffusion of slow neutrons // Phys. Rev., 1936, v.50(10), p. 899-928.

Амальди Э., Ферми Э. О поглощении и диффузии медленных нейтронов / Э. Ферми. Научные труды. Т.1. - М.: Наука. 1971, с. 686-740.

41. Марчук Г.И. Методы расчета ядерных реакторов. - М.: Госатомиздат, 1961. Marchuk G.I. Metody rascheta iadernykh reaktorov. - M.: Gosatomizdat, 1961.

42. Белл Д., Глесстон С. Теория ядерных реакторов. - М.: Атомиздат, 1974.

Bell G.I., Glasstone S. Nuclear reactor theory. - NY: Van Nostrand Reinhold, 1970.

43. Николаев М.Н., Рязанов Б.Г., Савоськин М.М., Цибуля А.М. Многогрупповое приближение в теории переноса нейтронов. - М.: Энергоатомиздат, 1984.

Nikolaev M.N., Riazanov B.G., Savoskin M.M., Tsibulia A.M. Mnogogruppovoe priblizhenie v teorii perenosa neitronov. - M.: Energoatomizdat, 1984.

44. Elsasser W.M. Mean absorption and equivalent absorption coefficient of a band spectrum // Phys. Rev., 1938, v.54(2), p.126-129.

45. Прудников А.П., Брычков Ю.А., Маричев О.И. Интегралы и ряды. Т.1. Элементарные функции. 2-е изд. - М.: Наука, 2002.

Prudnikov A.P., Brychkov Yu.A., Marichev O.I. Integrals and Series. V.1. Elementary Functions. Taylor and Francis, 2002.

46. Сеге Г. Ортогональные многочлены. - М.: Физматлит, 1962.

Szego G. Orthogonal polynomials. - Providence: Am. Math. Soc., 1939.

47. Li J., Li J. Angular anisotropy of group averaged absorption coefficient and its effect on the behavior of diffusion approach in radiative transfer // Quant. Spectrosc. and Rad. Transfer, 2009, v. 110(4), p. 293-299. 